\title{
Matricellular protein CCN3 mitigates abdominal aortic aneurysm
}

\author{
Chao Zhang, ${ }^{1,2}$ Dustin van der Voort, ${ }^{1}$ Hong Shi, ${ }^{1}$ Rongli Zhang, ${ }^{1}$ Yulan Qing, ${ }^{3}$ Shuichi Hiraoka, ${ }^{4}$ Minoru Takemoto, ${ }^{5}$ \\ Koutaro Yokote, ${ }^{5}$ Joseph V. Moxon, ${ }^{6}$ Paul Norman, ${ }^{7}$ Laure Rittié, ${ }^{8}$ Helena Kuivaniemi, ${ }^{9}$ C. Brandon Atkins, ${ }^{1}$ Stanton L. Gerson, ${ }^{3}$ \\ Guo-Ping Shi, ${ }^{10}$ Jonathan Golledge, ${ }^{6}$ Nianguo Dong, ${ }^{2}$ Bernard Perbal, ${ }^{11}$ Domenick A. Prosdocimo, ${ }^{1}$ and Zhiyong Lin ${ }^{1}$
}

\begin{abstract}
'Case Cardiovascular Research Institute, Case Western Reserve University School of Medicine, Harrington Heart and Vascular Institute, University Hospitals Case Medical Center, Cleveland, Ohio, USA. 2Department of Cardiovascular Surgery, Union Hospital, Tongji Medical College, Huazhong University of Science and Technology, Wuhan, China. ${ }^{3}$ Division of Hematology and Oncology, University Hospitals Case Medical Center, Case Western Reserve University School of Medicine, Cleveland, Ohio, USA. ${ }^{4}$ Department of Systems BioMedicine, National Research Institute for Child Health and Development, Tokyo, Japan. ${ }^{5}$ Department of Medicine, Chiba University Graduate School of Medicine, Chiba, Japan. ${ }^{6}$ The Vascular Biology Unit, Queensland Research Centre for Peripheral Vascular Disease, School of Medicine and Dentistry, James Cook University, and The Department of Vascular and Endovascular Surgery, Townsville Hospital, Townsville, Queensland, Australia. ' 'School of Surgery, University of Western Australia, Perth, Western Australia, Australia. ${ }^{8}$ Department of Dermatology, University of Michigan, Ann Arbor, Michigan, USA. ${ }^{9}$ Division of Molecular Biology and Human Genetics, Department of Biomedical Sciences, Faculty of Medicine and Health Sciences, Stellenbosch University, Tygerberg, South Africa. ${ }^{10}$ Department of Medicine, Brigham and Women's Hospital and Harvard Medical School, Boston, Massachusetts, USA. "University of Paris Diderot - Paris 7, Paris, France.
\end{abstract}

\begin{abstract}
Abdominal aortic aneurysm (AAA) is a major cause of morbidity and mortality; however, the mechanisms that are involved in disease initiation and progression are incompletely understood. Extracellular matrix proteins play an integral role in modulating vascular homeostasis in health and disease. Here, we determined that the expression of the matricellular protein CCN3 is strongly reduced in rodent AAA models, including angiotensin II-induced AAA and elastase perfusion-stimulated AAA. CCN3 levels were also reduced in human AAA biopsies compared with those in controls. In murine models of induced AAA, germline deletion of $\mathrm{Ccn} 3$ resulted in severe phenotypes characterized by elastin fragmentation, vessel dilation, vascular inflammation, dissection, heightened ROS generation, and smooth muscle cell loss. Conversely, overexpression of CCN3 mitigated both elastase- and angiotensin II-induced AAA formation in mice. BM transplantation experiments suggested that the AAA phenotype of CCN3-deficient mice is intrinsic to the vasculature, as AAA was not exacerbated in WT animals that received CCN3-deficient BM and WT BM did not reduce AAA severity in CCN3-deficient mice. Genetic and pharmacological approaches implicated the ERK1/2 pathway as a critical regulator of CCN3-dependent AAA development. Together, these results demonstrate that CCN3 is a nodal regulator in AAA biology and identify CCN3 as a potential therapeutic target for vascular disease.
\end{abstract}

\section{Introduction}

Disruption of aortic homeostasis arising from genetic defects or exposure to environmental risk factors leads to localized abnormal widening of the aorta, a degenerative disease state termed aortic aneurysm that can affect the length of the aorta from its root to the abdominal segment $(1,2)$. Experimental studies reveal that aortic aneurysm is associated with compromised smooth muscle function, extracellular matrix (ECM) degradation, and increased vascular inflammation associated with leukocyte infiltration (2-6). This pathologic aortic milieu culminates with weakening of the vessel wall, a central characteristic of aortic aneurysm disease. Abdominal aortic aneurysm (AAA) is usually asymptomatic; however, rupture of an aortic aneurysm constitutes an important cause of mortality in the elderly that causes more than 15,000 deaths annually in the United States (WISQARS Leading Causes of Death Reports (19992013); http://webappa.cdc.gov/sasweb/ncipc/leadcaus10_us.html). Despite the risk of mortality associated with AAA, there are no cur-

Conflict of interest: The authors have declared that no conflict of interest exists. Submitted: April 16, 2015; Accepted: January 28, 2016.

Reference information: J Clin Invest. 2016;126(4):1282-1299. doi:10.1172/JCI82337. rent drug therapies that have been shown to limit AAA development or progression (7). Currently, timely open or endovascular surgical repair remains the only form of treatment (8). Therefore, the elucidation of the cellular and molecular basis for this disease is imperative for the development of novel pharmacologic therapies and management strategies.

In addition to providing structural support to the vasculature, the ECM is indispensable in modulating cellular signaling and vessel performance $(9,10)$. This ECM milieu dictates organismal health, while perturbation of ECM homeostasis is implicated in the development of pathological conditions, including atherosclerosis, restenosis, and AAA, with ECM proteins, such as collagen, elastin, and integrins, serving an essential role in mediating disease progression (11). However, despite considerable progress, identification of the molecular mechanisms underlying the ECMdependent modulation of vascular wall homeostasis in AAA initiation and progression remains incomplete.

CCN (Cyr61, Ctgf, Nov) proteins are a group of secreted ECMassociated, partially identical cysteine-rich proteins that mediate diverse biologic functions (12-14). Although accumulating evidence demonstrates that the CCN gene family is critical to cell 
A

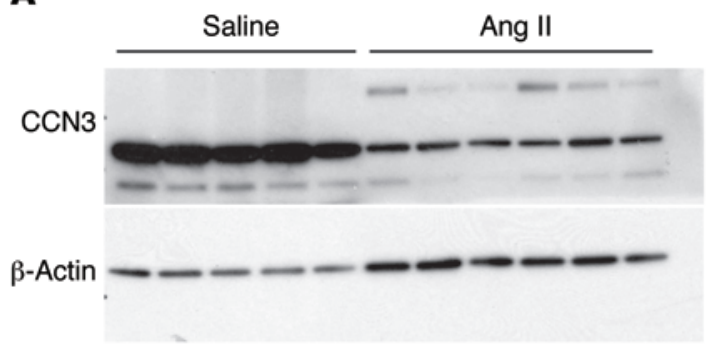

C

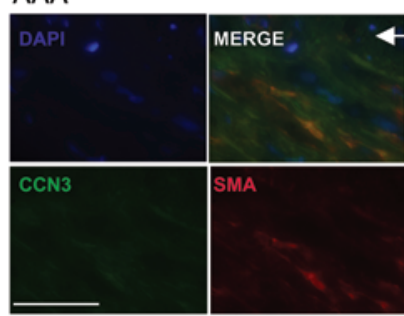

Non-AAA

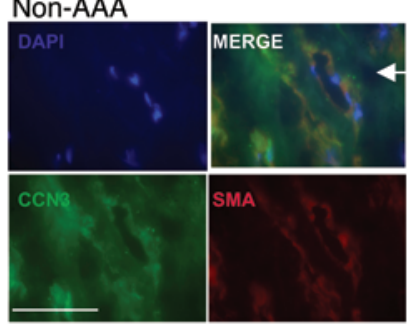

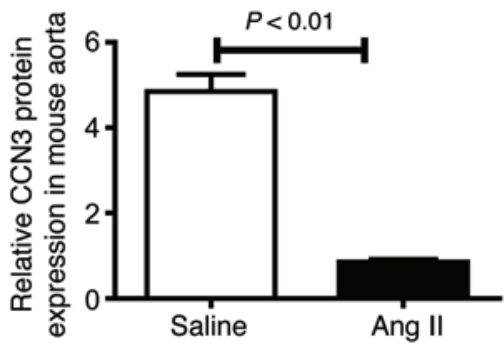

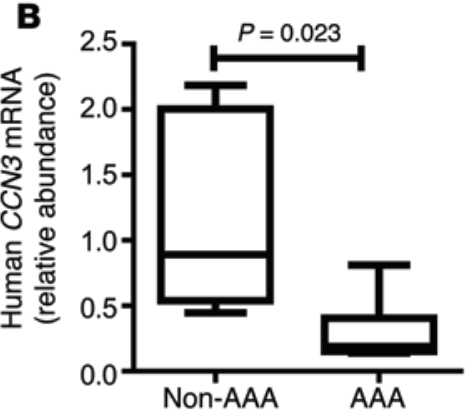

D
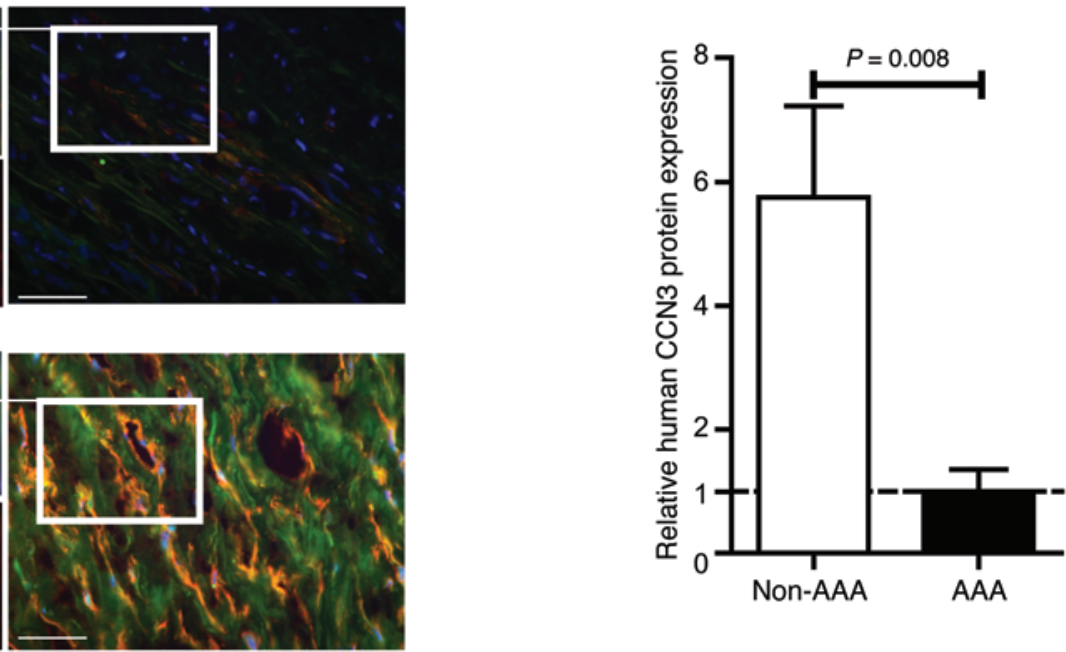

Figure 1. CCN3 expression is reduced in AAA. (A) Western blot of CCN3 protein in mouse abdominal aorta tissue from Apoe-null mice infused with saline or Ang II for 4 weeks. $n=5-6$. (B) CCN3 mRNA expression from human abdominal aorta tissue without and with AAA, $n=5-6$ per group, Mann-Whitney $U$ test. Representative image of CCN3 protein expression by immunofluorescence staining (C) and quantification from control human nonaneurysmal infrarenal aorta and AAA (D). Scale bars: $50 \mu \mathrm{m}$. Red, SMA; green, CCN3; orange, overlay of SMA (red) and CCN3 (green). Mann-Whitney $U$ test.

growth and differentiation, the physiological importance of CCN proteins in the vasculature remains largely undefined. A member of this gene family, CCN3 (also known as NOV), was originally isolated from nephroblastoma tissue in newborn chicks infected with the MAV-1 avian retrovirus (15). Recent studies suggest CCN3 regulates vascular function $(16,17)$. However, whether alterations of CCN3 expression or function play a causal role in AAA disease remains unknown. In the current study, we utilized 2 established models of aortic aneurysm (angiotensin II [Ang II] infusion and elastase perfusion) to address whether $\mathrm{CCN} 3$ is required to regulate AAA pathobiology. Herein, we provide evidence that CCN3 serves as a negative regulator of AAA initiation and progression by ameliorating inflammatory cell infiltration, smooth muscle cell (SMC) apoptosis, and elastin degradation through a mechanism involving the ERK1/2 pathway. Our studies offer insights regarding the regulation of AAA formation by CCN3 and provide the foundation for the development of future therapeutics for this disease.

\section{Results}

Reduction of CCN3 in rodent and human AAA tissues. Previous studies suggest that CCN3 is expressed in SMC and endothelial cells (ECs) (16-18). To gain a broader understanding, we first assessed CCN3 expression in the vasculature of WT mice by immunohistochemistry. As shown in Supplemental Figure 1 (supplemental mate- rial available online with this article; doi:10.1172/JCI82337DS1), CCN3 expression was detected in both arterial and venous SMCs from multiple vascular beds. To gain initial insights into the function of CCN3 in vascular disease, we assessed endogenous CCN3 expression in the abdominal aortic tissues from experimental AAA models. This included Ang II infusion in apolipoprotein E-deficient (Apoe-null) mice and elastase perfusion in C57BL/6 mice, both of which share some similarities to human AAA disease. As shown in Figure 1A, CCN3 protein was markedly reduced in rodent abdominal aorta tissues following 4 weeks of Ang II infusion. Moreover, infusion of Ang II caused a time-dependent decrease of CCN3 expression (Supplemental Figure 2A). Similar findings were obtained in an elastase perfusion model (Supplemental Figure 2B). We next assessed CCN3 expression in infrarenal aortic biopsies taken from AAA patients and nonaneurysmal controls. Concordant with findings in mouse AAA tissues, a dramatic reduction of CCN3 mRNA expression was seen in human AAA tissues compared with control aorta tissues (Figure 1B), findings that were confirmed in a previously published microarray data set (19). Finally, CCN3 protein was reduced in the AAA tissues, as revealed by immunostaining (Figure 1, C and D). Of note, costaining of smooth muscle $\alpha$-actin (SMA) and CCN3 suggested a reduction of CCN3 in SMCs (Figure 1C). Collectively, these data demonstrate that CCN3 is expressed in the aorta and that its expression is reduced in AAA. 
A

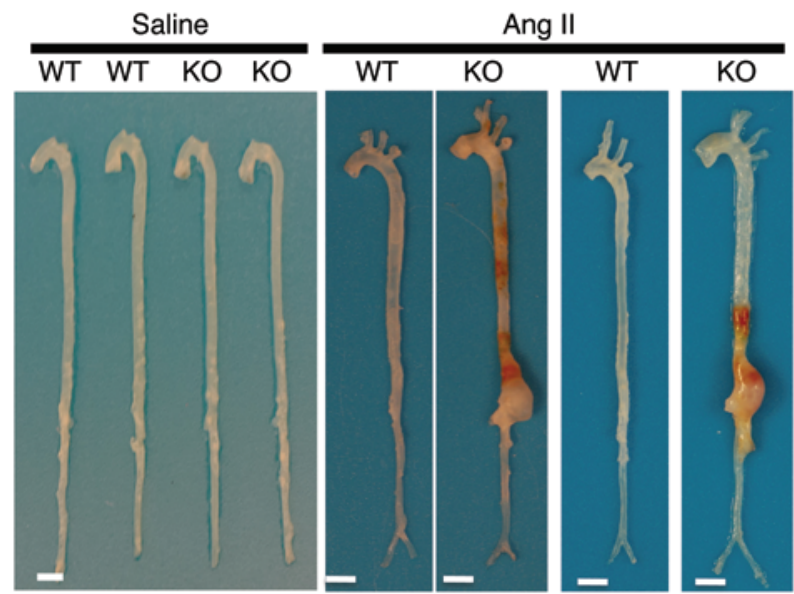

C
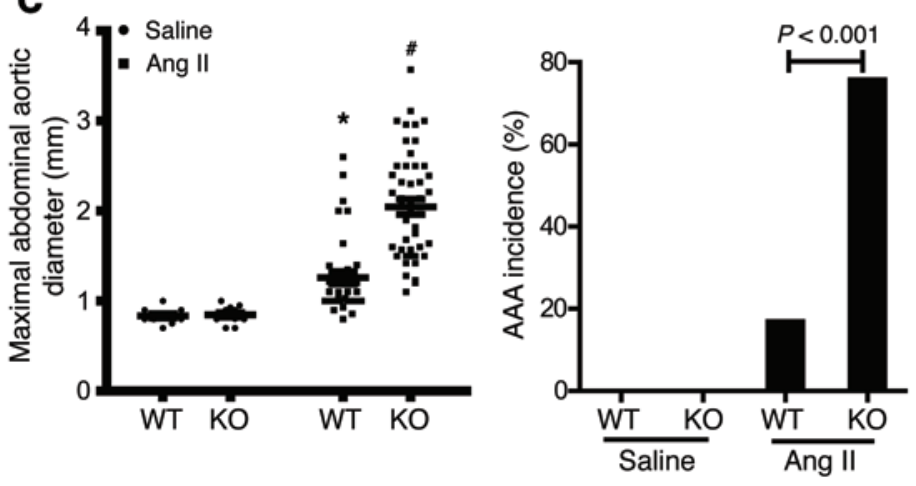

B

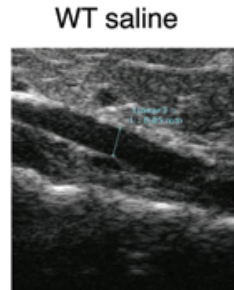

KO saline

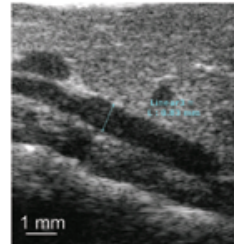

D

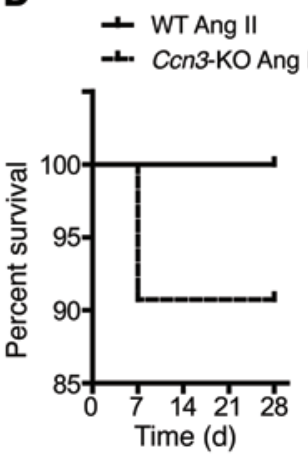

WT Ang II

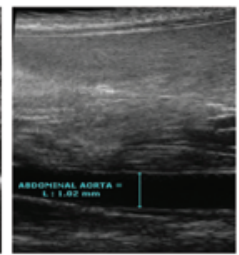

KO Ang II
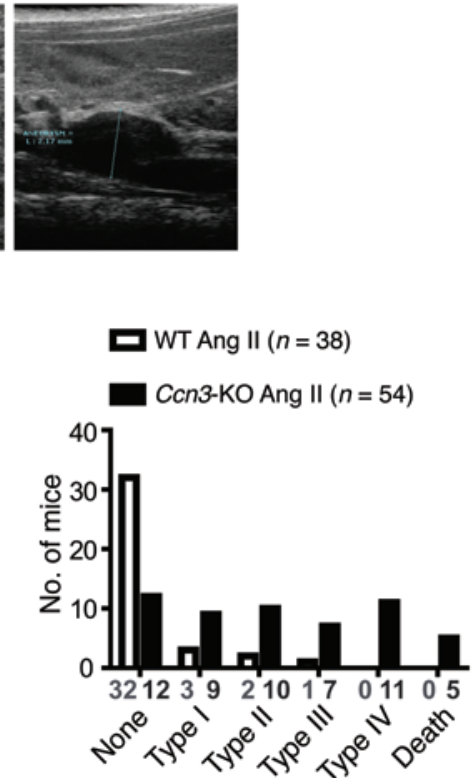

E

F WT saline

KO saline

Saline<smiles>c1ccccc1</smiles>

Ang II
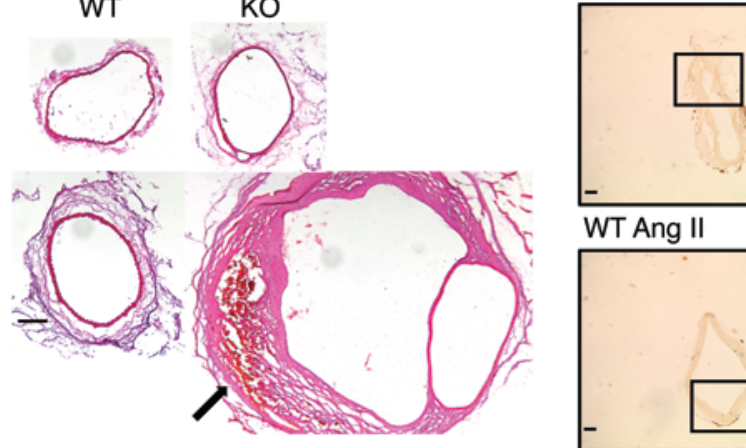

G

WT

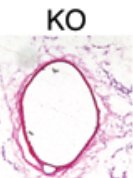

KO
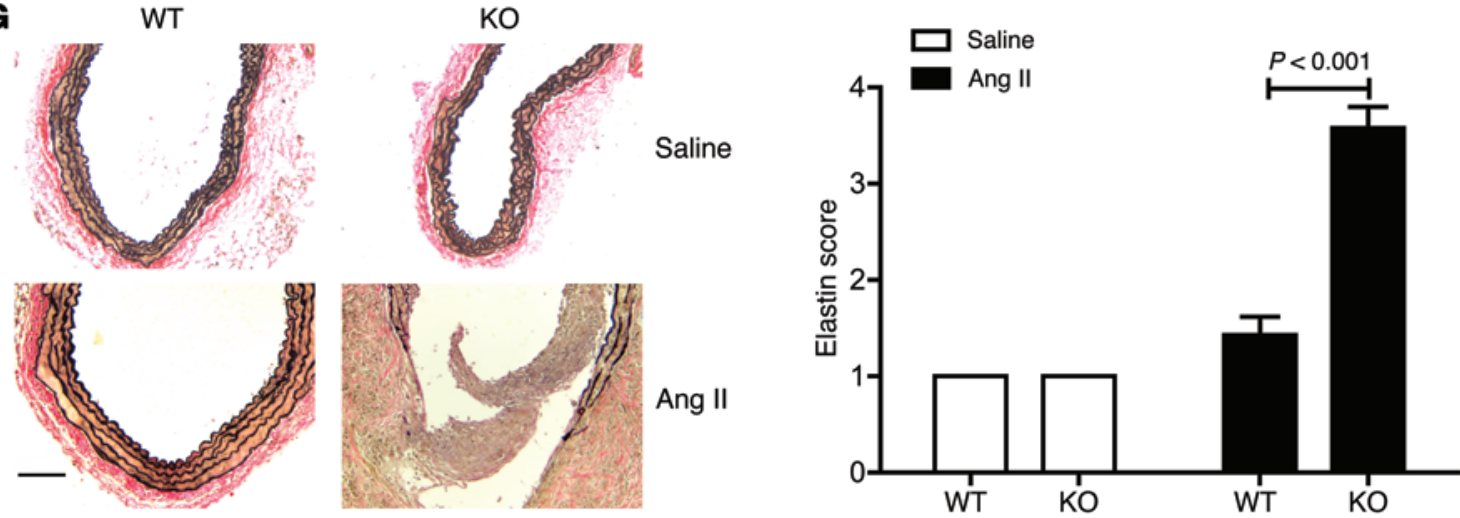
Figure 2. CCN3 deficiency augments experimentally induced AAA in mice. (A) Representative image of whole aortae from WT and CCN3-deficient mice following 4 weeks of saline or Ang II infusion. Scale bars: $2.5 \mathrm{~mm}$. (B) Representative ultrasound images from WT and CCN3-deficient mice following 4 weeks of saline or Ang II infusion. (C) Maximal abdominal aortic diameter (left panel), AAA incidence (right panel). Saline treatment, WT, $n=11 ; \mathrm{KO}, n=13$. Ang II treatment, WT, $n=38$; KO, $n=49$. * $P<0.05$, WT saline versus WT, Ang II; ${ }^{P}<0.001$, WT Ang II versus KO Ang II. (D) KaplanMeier survival curve (left panel, $P=0.06$, log-rank test) and AAA classification (right panel) in WT versus CCN3-deficient aortae following 4 weeks of saline or Ang II infusion. (E) H\&E staining of abdominal aorta samples from WT and CCN3-deficient aortas following 4 weeks of saline or Ang II infusion. Arrow indicates intramural hematoma. Scale bar: $100 \mu \mathrm{m}$. (F) Representative TER119 staining in WT versus CCN3-deficient aortae following 28 days of saline or Ang II infusion. Scale bars: $50 \mu \mathrm{m}$. (C) Elastin integrity grading in WT versus CCN3-deficient aortae following 4 weeks of saline or Ang II infusion. Data are presented as mean \pm SEM. Saline group, WT, $n=5$; $\mathrm{KO}, n=7$. Ang II group, WT, $n=7$; KO, $n=12$. WT, $\mathrm{CCn}^{+/+} ; \mathrm{KO}, \mathrm{Ccn3}^{-/-}$. Scale bar: $50 \mu \mathrm{m}$. For comparing AAA incidence, $\chi^{2}$ test was used. In all others, 2-way ANOVA followed by Bonferroni's post-hoc correction was used.

Genetic ablation of $C C N 3$ results in aggressive AAA formation. Given the observation that $\mathrm{CCN} 3$ expression is reduced in AAA, we hypothesized that CCN3 deficiency may exacerbate AAA formation. To test this, we first carried out CCN3 loss-of-function studies using a model of AAA formation that involves elastase perfusion $(20,21)$. CCN3-deficient mice are healthy, with no alterations in growth, reproduction, or tissue morphology (17). In these studies, 8- to 10-week-old male CCN3-deficient and
WT littermate controls (both on C57BL/6 backgrounds) were subjected to elastase or normal saline perfusion. Consistent with our hypothesis, CCN3-deficient animals were more susceptible to abdominal aortic dilation following elastase perfusion (Supplemental Figure 3, A and B). To quantify the changes in aorta dilation following elastase perfusion, the external infrarenal abdominal aortic diameter was measured 14 days after elastase perfusion. As shown in Supplemental Figure 3C, at the end of the perfusion, the mean external infrarenal abdominal aortic diameter was $0.98 \pm 0.04 \mathrm{~mm}$ in WT mice, which was significantly smaller than that in the CCN3-deficient mice $(1.29 \pm 0.09 \mathrm{~mm}$, $P=0.006$, Supplemental Figure 3C). Concordant with this, the mean maximal increase in infrarenal aortic diameter following elastase infusion in the CCN3-deficient mice was significantly greater than in the WT controls (WT: $86.01 \% \pm 7.67 \%$, versus KO: $140.60 \% \pm 16.47 \% ; P=0.004$, Supplemental Figure $3 \mathrm{C}$ ).

We next sought to confirm the above findings in a second model of AAA formation that involved Ang II infusion, a model that recapitulated some human AAA pathological characteristics, including inflammatory response and reactive oxygen species (ROS) generation within the arterial wall (21-23). Age-matched male WT and CCN3-deficient mice were subjected to either saline or Ang II (1000 ng/ $\mathrm{kg} / \mathrm{min})$ infusion for 28 days. With saline infusion, we observed no gross differences in morphology between the aortas of WT and CCN3-deficient mice. Additionally, electron microscopy studies showed no obvious differences in aortic elastin density between WT and CCN3-deficient mice
Figure 3. CCN3 deficiency promotes a proinflammatory response. Representative images of immunohistochemical staining for macrophages (MAC3) (A), T cells (CD3) (B), MCP1 (C), and VCAM1 (D) in WT versus CCN3-deficient aortae following 28 days of Ang II infusion; quantification shown in the right panels. Saline, $n=3$; Ang II, $n=5-6$. Two-way ANOVA followed by Bonferroni's test. Scale bars: $100 \mu \mathrm{m}$.

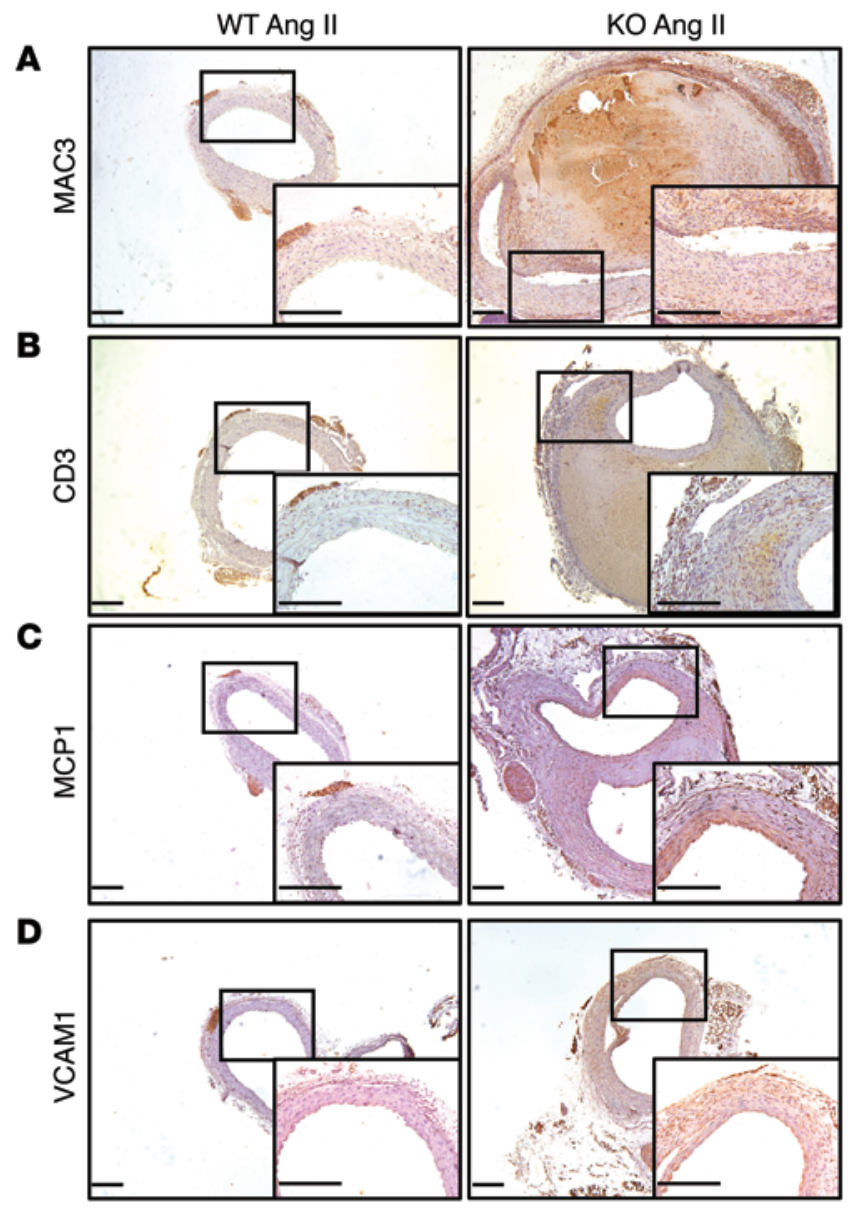

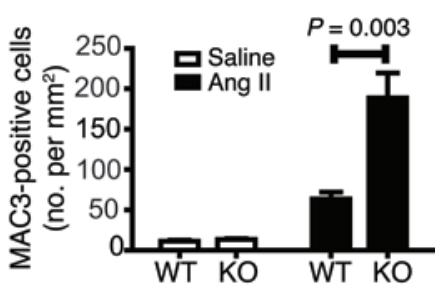
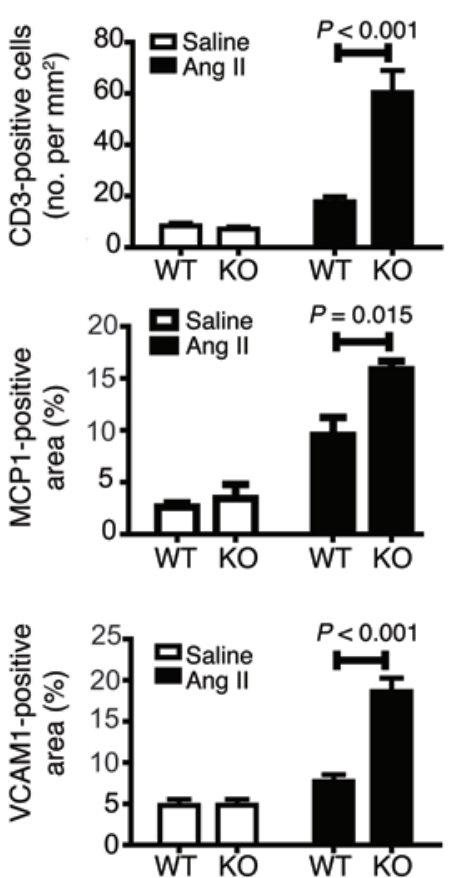
A WT Ang II KO Ang II
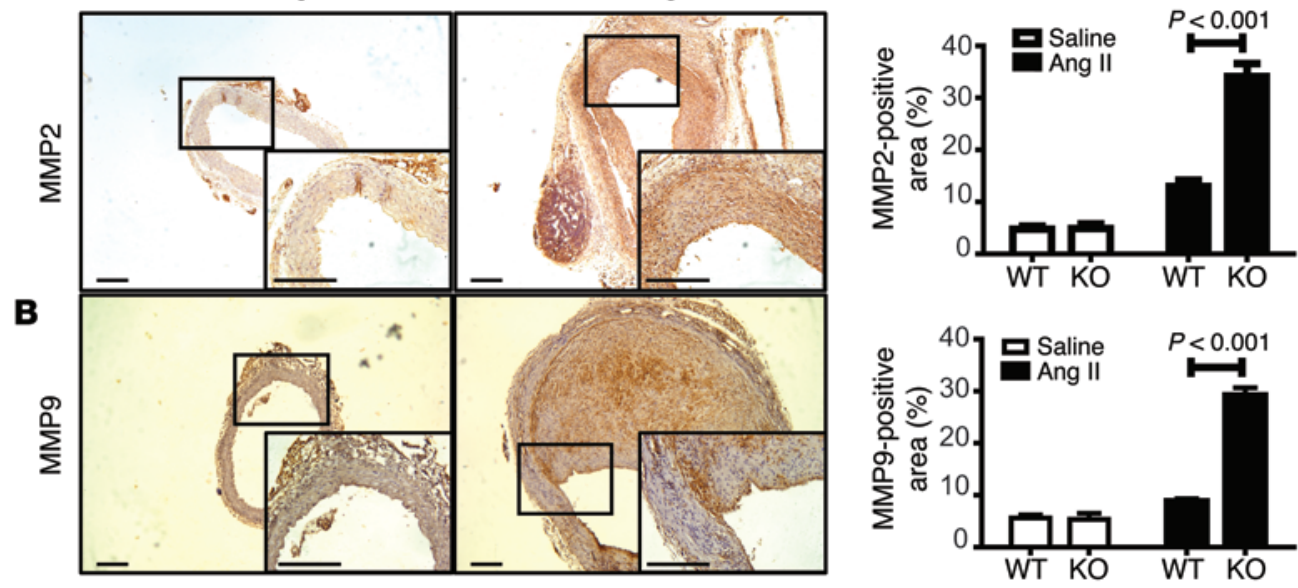

C
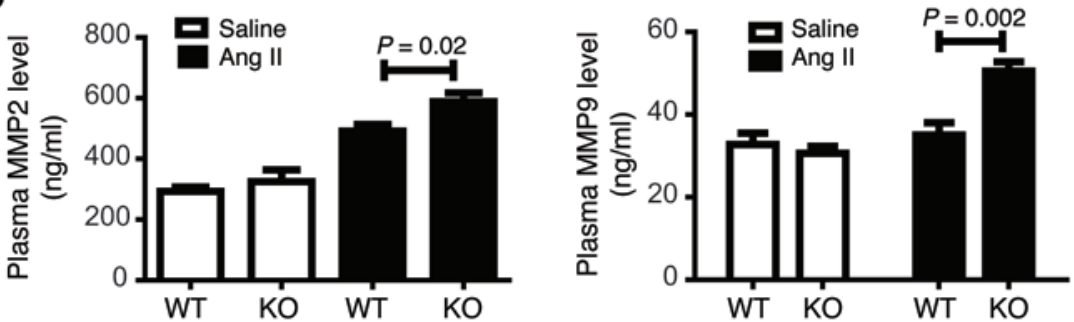

D
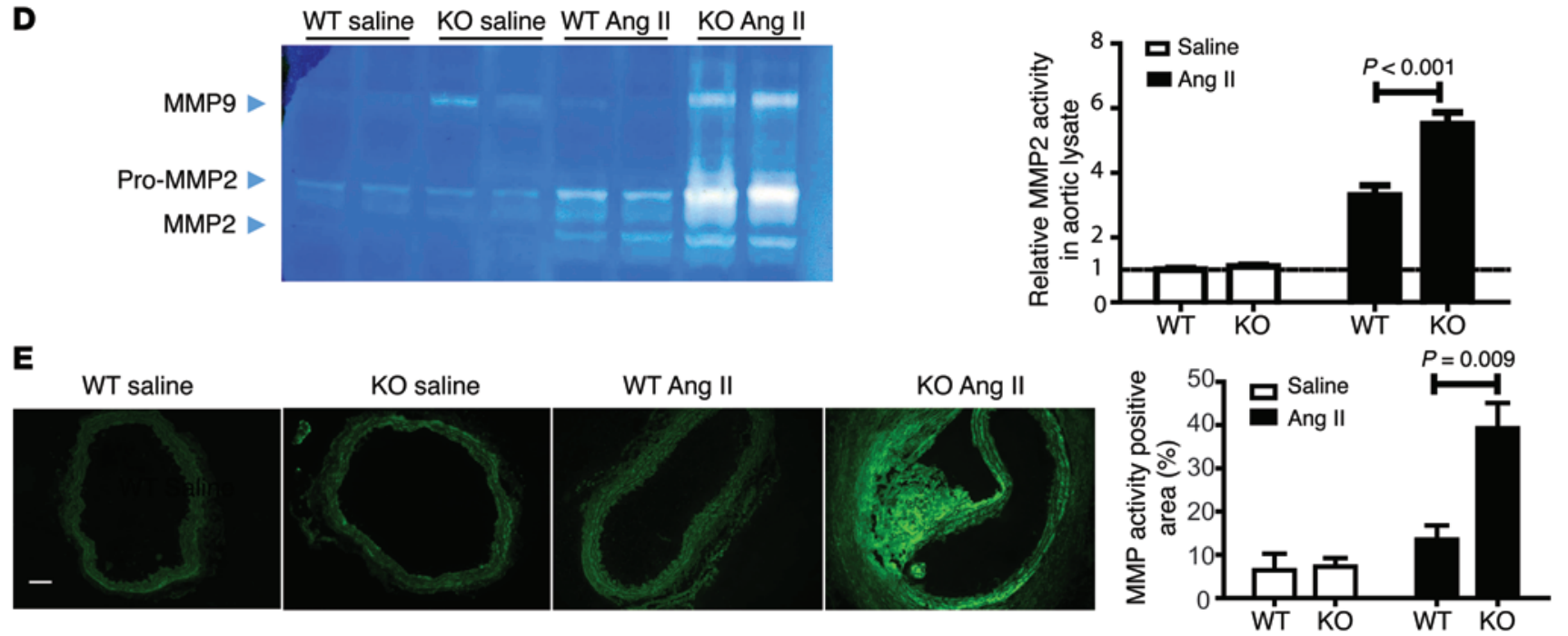

Figure 4. CCN3 deficiency promotes MMP production in CCN3-deficient aortas following 4 weeks of Ang II infusion. (A) Immunohistochemical staining for MMP2 and (B) MMPg; quantification shown in the right panel. Saline, $n=3$; Ang II, $n=5-7$. Representative images for saline-infused group are shown in Supplemental Figure 7. (C) Plasma MMP2 and MMPg levels analyzed by ELISA ( $n=3$ for saline group; WT Ang II, $n=6$; KO Ang II, $n=12$ ). (D) MMP activity in abdominal aortic protein lysates by zymography (saline, $n=3$; Ang II, $n=8$ ). Pro-MMP2, full-length MMP2. (E) In situ MMP activity, saline, $n=3$; Ang II, $n=6$. Two-way ANOVA followed by Bonferroni's test. Scale bars: $100 \mu \mathrm{m}$.

(Supplemental Figure 4). Moreover, CCN3-deficient aortas demonstrated no medial hypertrophy and there was no significant difference in blood pressure between WT and CCN3-deficient mice under basal and following Ang II infusion (ref. 17 and data not shown). Additionally, there was no difference in the lipid profile of WT and CCN3-deficient mice after 4 weeks of Ang II infusion (Supplemental Figure 5). However, in response to 28 days of Ang II infusion, the aortas from CCN3-deficient mice displayed exquisite sensitivity, findings that were in agreement with the elastase perfusion model. CCN3-deficient mice infused with Ang II for
28 days developed generalized aortic dilation (Figure 2, A and C). The mean maximal suprarenal abdominal aortic diameters of aortas in the CCN3-deficient mice were significantly greater than those of the WT controls (WT: $1.25 \pm 0.07 \mathrm{~mm}, n=38$, versus CCN3-deficient: $2.04 \pm 0.08 \mathrm{~mm}, n=49 ; P<0.001)$. Adopting a binary scoring system, more than $70 \%$ of $\mathrm{Ccn3}^{-/-}$mice developed AAAs, while less than $16 \%$ of WT mice developed AAA $(P<$ 0.001 , Figure 2C). The AAA phenotype of CCN3-deficient mice after Ang II infusion was also manifested by ultrasound imaging that confirmed the finding that CCN3-deficient mice had a larger 
A

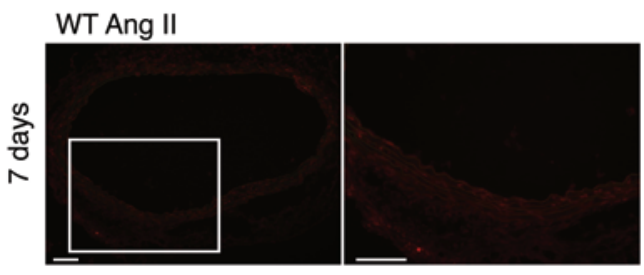

B

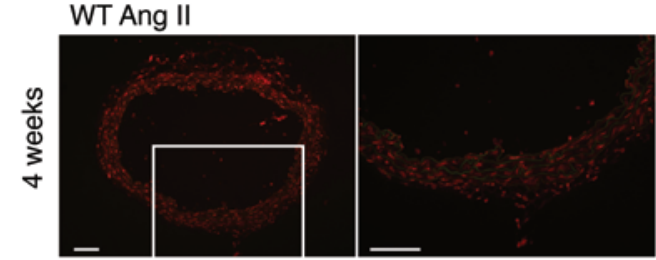

C

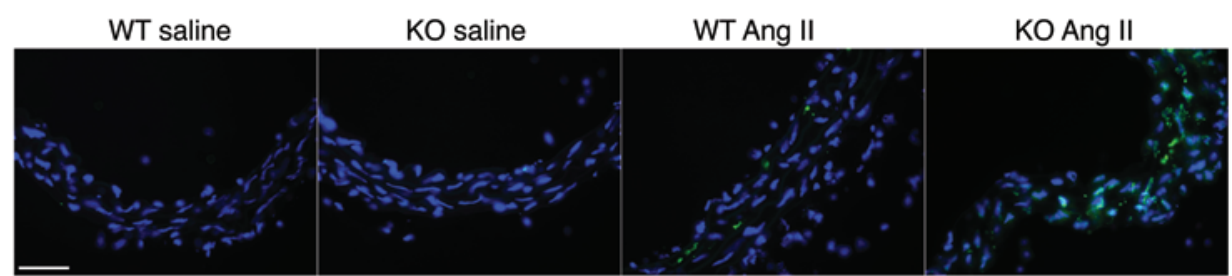

D
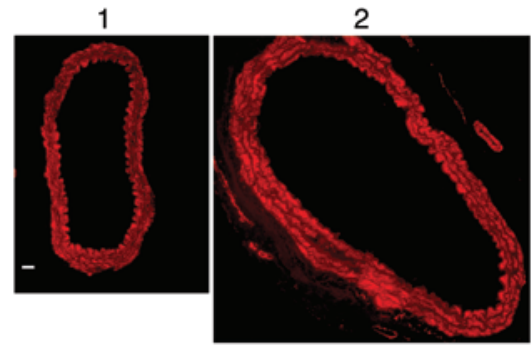

KO Ang II

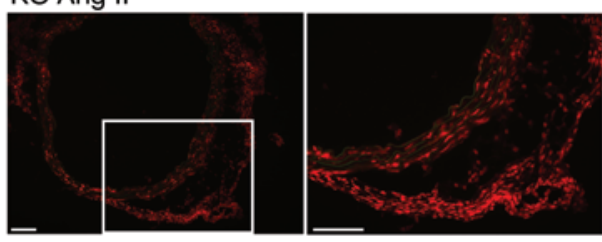

KO Ang II
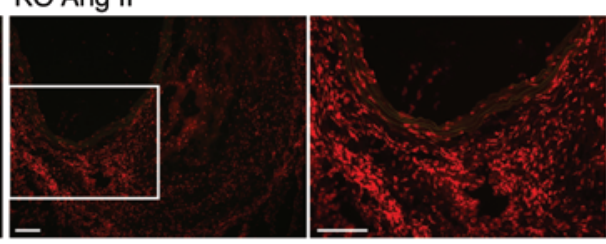

KO Ang

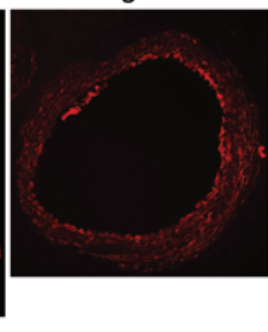

4

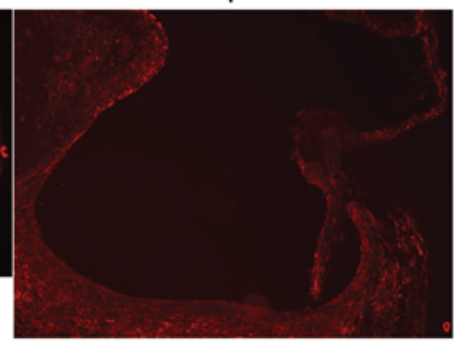

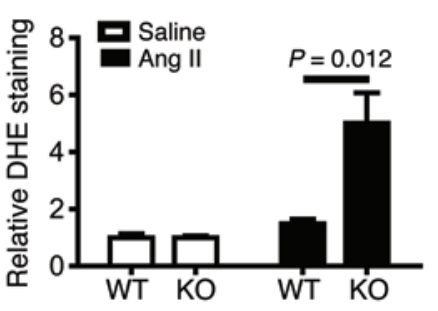
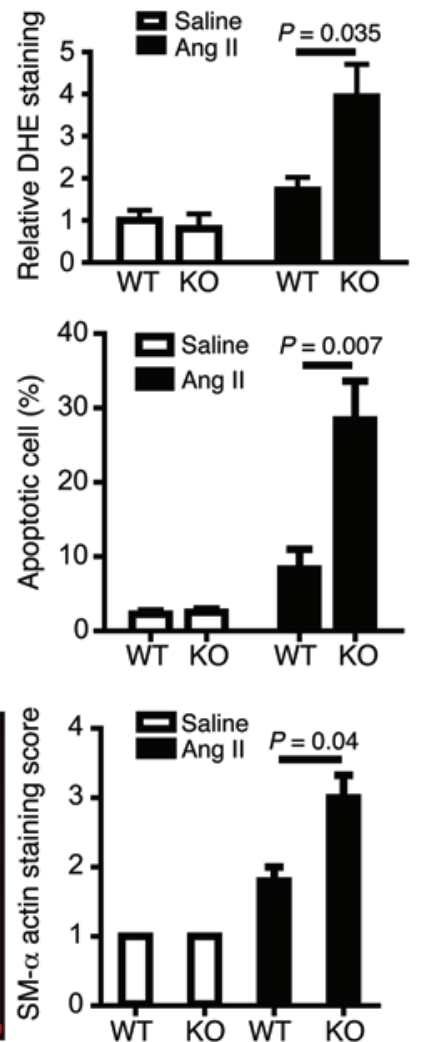

Figure 5. CCN3 deficiency promotes ROS generation and loss of SMCs in CCN3-deficient aortas following Ang II infusion. (A) ROS generation 7 days after Ang II infusion assessed by DHE staining (saline, $n=3$; Ang II, $n=5$ ). (B) ROS generation 4 weeks after Ang II infusion (saline, $n=3$; Ang II, $n=5-6$ ). (C) SMC TUNEL staining following 7 days of Ang II treatment in WT versus CCN3-deficient aortas (saline, $n=3$; Ang II, $n=5-6$ ). Green, TUNEL positive; blue, DAPI nuclear staining. (D) SMC loss assessed by SMA staining 4 weeks after Ang II infusion in WT versus CCN3-deficient aortas (saline, $n=3$; Ang II, $n=5-8$ ). Grade 1, intact media, no SMC loss; grade 2, modest loss of SMC; grade 3, severe loss of SMC; grade 4, rupture of media, almost no SMC left. Two-way ANOVA followed by Bonferroni's test. Scale bars: $50 \mu \mathrm{m}$.

abdominal aorta (Figure 2B). In addition, 5 CCN3-deficient mice died of aortic rupture, while no such deaths were observed in the WT group (Figure 2D). We further classified AAA formation in WT and CCN3-deficient mice based on the severity of AAA as suggested by Daugherty and colleagues (24). Using this classification system, CCN3-deficient mice developed more severe AAAs than WT mice (Figure 2D).

To assess the histological features of the AAAs, we performed $\mathrm{H} \& \mathrm{E}$ and elastin staining. Histologic examination revealed intramural hematoma formation in the aneurysmal tissues. As shown in Figure 2, E and F, the presence of red blood cells within the aortic wall of CCN3-deficient mice was evidenced by H\&E staining as well as immunostaining for TER119 (marker for red blood cells). Additionally, the aortas of CCN3-deficient mice that received Ang II were characterized by severe disruption of medial architecture with prominent elastin degradation, which is another key feature of AAA. Profoundly disrupted and fragmented elastic lamellae were seen in the aortas of CCN3-deficient mice, as demonstrated by Verhoeff-van Gieson staining (Figure 2G). Similarly, dramatic elastin degradation was observed in the aortas of elastaseperfused CCN3-deficient mice (Supplemental Figure 6A).

Taken together, these data suggest that CCN3-deficient aortas are highly sensitive to experimentally induced AAA formation (by elastase perfusion or Ang II infusion) and develop aortopathy with markedly increased frequency of AAA marked by deteriorated ECM, suggesting a protective role of CCN3 against AAA formation.

Loss of CCN3 exacerbates vascular inflammation and MMP activities. As AAA is typically accompanied by vascular inflammation, we next sought to evaluate whether Ang II infusion gives rise to a heightened state of vascular inflammation in CCN3deficient mice. One of the hallmark features of both human AAA and Ang II-induced AAA in rodents is the accumulation of inflammatory infiltrates in both the adventitia and media layers 

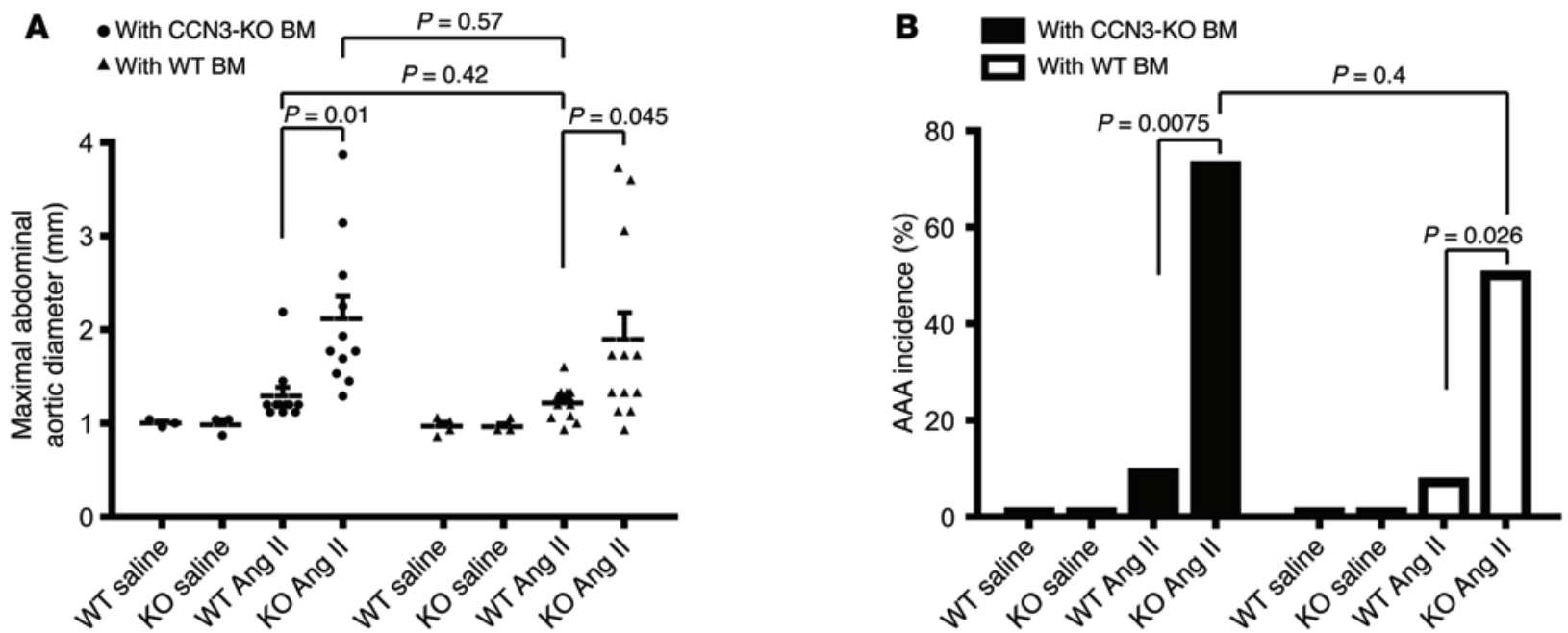

C

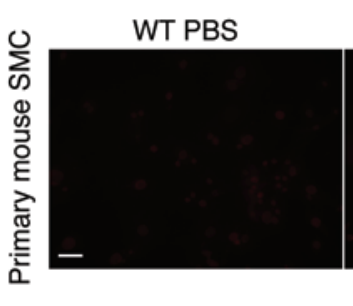

KO PBS

WT Ang II

KO Ang II

D
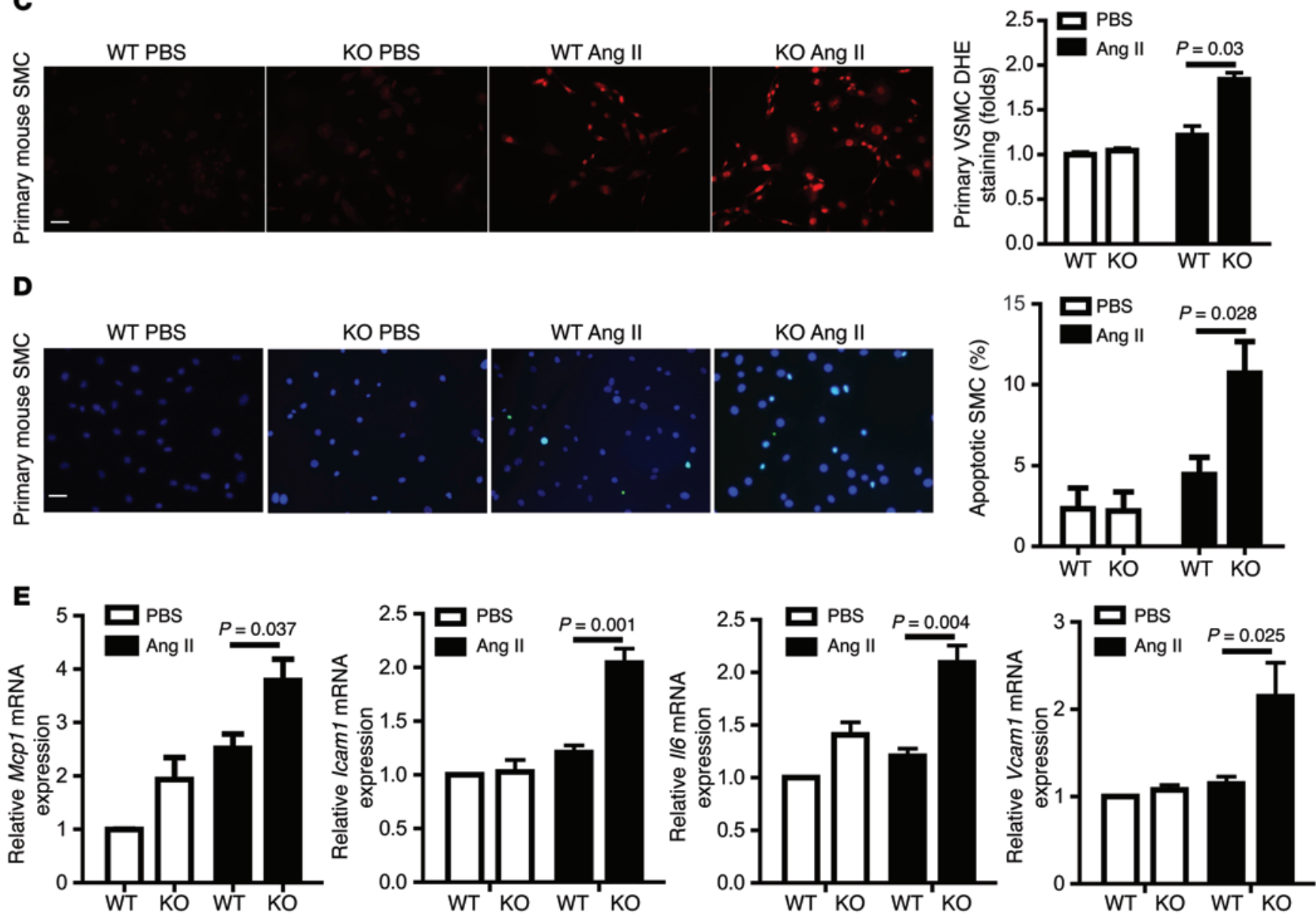

Figure 6. The AAA phenotype in CCN3-deficient mice is intrinsic to the vascular wall. (A) Maximal external abdominal aortic diameter and (B) AAA incidence of BMT mice following Ang II infusion. $n=3-4$ for saline group; $n=11-14$ for Ang II group. (C) ROS production (assessed by DHE staining) in SMCs following Ang II treatment in WT versus CCN3-deficient mice. $n=6$ per group. (D) TUNEL staining in cultured SMCs from WT and CCN3-deficient aortas following Ang II treatment. $n=5$ independent experiments. (E) Mcp1, Icam1, IIG, and Vcam1 mRNA expression in WT versus CCN3-deficient SMCs in the absence or presence of Ang II; $n=4$ per group. Scale bars: $50 \mu \mathrm{m}$. Two-way ANOVA followed by Bonferroni's post-hoc correction was used.

(25). As such, we first examined inflammatory cell infiltration. In saline-perfused controls, there was minimal inflammatory response (Supplemental Figure 7). However, as expected, Ang II infusion led to the accumulation of inflammatory cells within the aortas of mice, as assessed by MAC3 (macrophage) and CD3
(T cell) staining (Figure 3, A and B). A more profound inflammatory infiltrate was observed in CCN3-deficient mice (Figure 3, A and $\mathrm{B})$. To gain further insights into the inflammatory response, immunostaining studies were performed to assess the expression of several key inflammatory molecules implicated in AAA, 

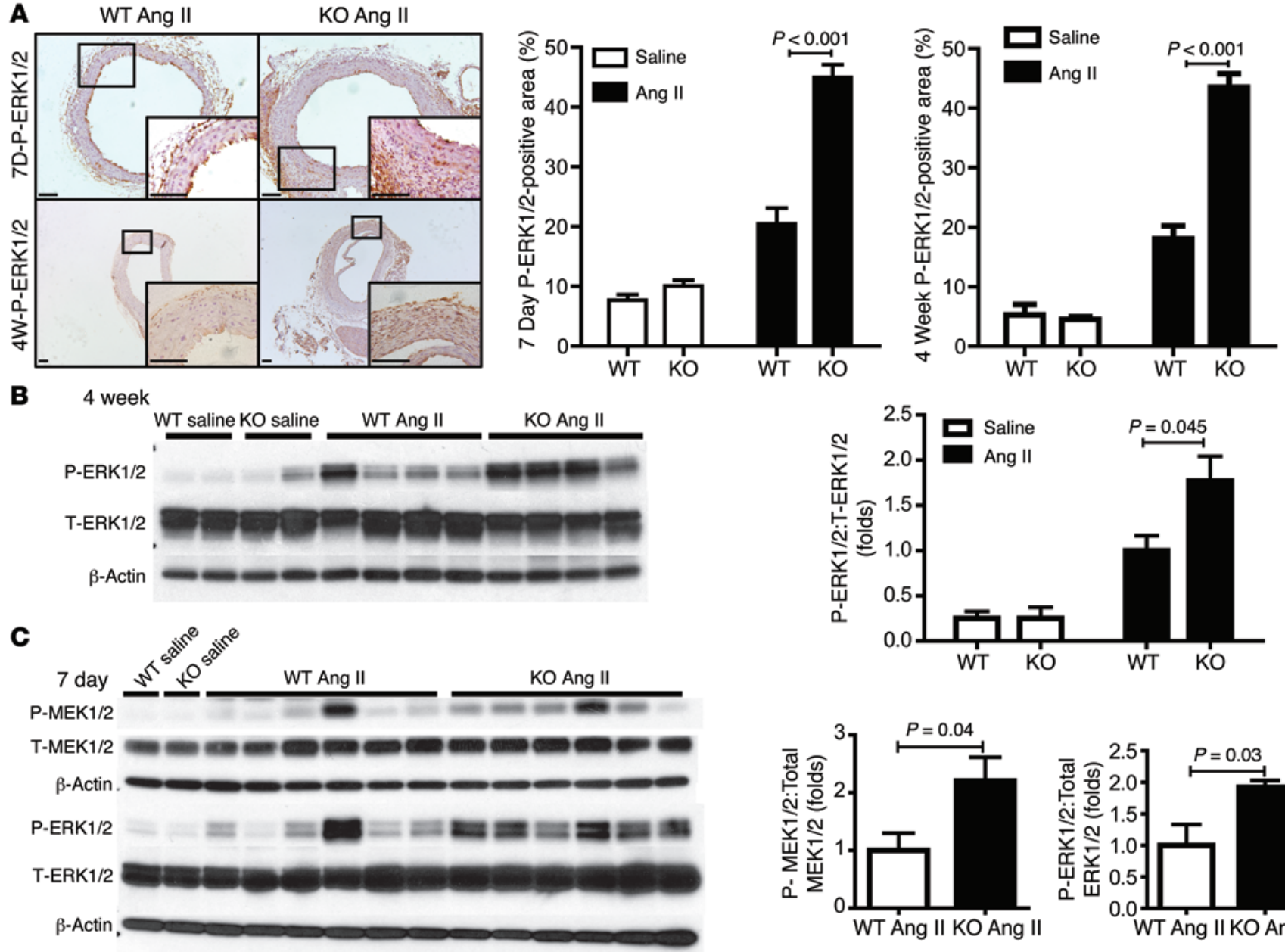

D
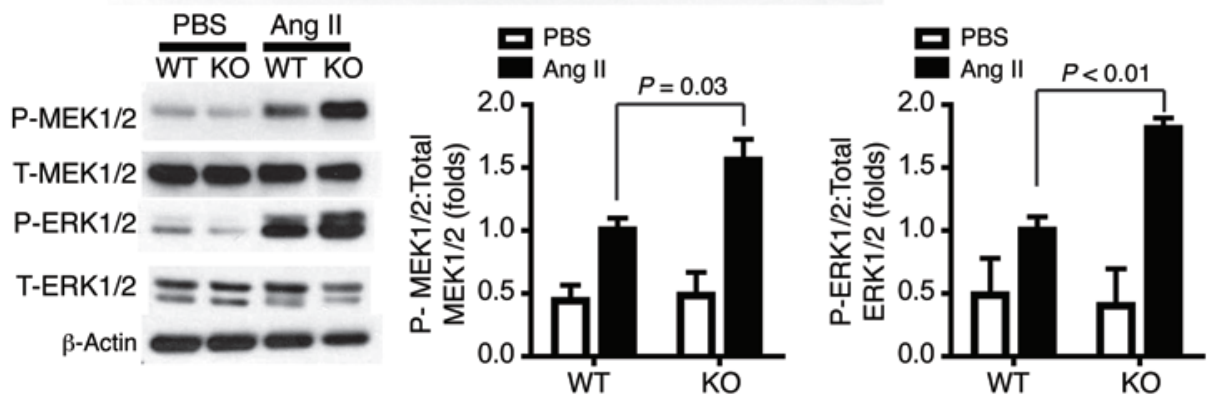

Figure 7. ERK1/2 activation by Ang II is increased in CCN3-deficient aorta. (A) Immunohistochemistry assessment of ERK1/2 activation in CCN3-deficient aortae in response to Ang II treatment (7 days and 4 weeks). Representative images are shown on the left; results are quantified on the right. $n=3-5$ per group. Scale bars: $100 \mu \mathrm{m}$. (B) Western blot of phospho-ERK1/2 in aortae following saline or Ang II treatment ( 4 weeks). $n=4$ for saline group; $n=6-8$ for Ang II group. (C) Western blot of phospho-ERK1/2 and phospho-MEK1/2 in aortae following saline or Ang II treatment ( 7 days). $n=4$ for saline group, $n=6-8$ for Ang II group. (D) Western blot of phospho-ERK1/2 and phospho-MEK1/2 in aortic SMCs following Ang II (30 minutes) treatment. $n=3$ for WT, $n=4$ for KO. T-ERK1/2, total ERK1/2; T-MEK1/2, total MEK1/2. Two-way ANOVA followed by Bonferroni's post-hoc correction was used.

including monocyte chemoattractant protein-1 (MCP-1) and VCAM-1. Consistent with the hyperinflammatory cell migration data, substantially greater VCAM-1 and MCP-1 staining was observed in the aortas of CCN3-deficient mice after Ang II infusion (Figure 3, C and D). In addition, we performed quantitative reverse-transcription PCR (qRT-PCR) experiments using suprarenal aortic segments. The mRNA expression of Mcp1 and Vcam1 was significantly increased in the aortas of CCN3-deficient mice receiving Ang II compared with controls (Supplemental Fig- ure $8 \mathrm{~A})$. We observed a similar enhancement of inflammatory response to elastase perfusion in the aortas of $\mathrm{CCN} 3$-deficient mice (MAC3 staining) (Supplemental Figure 6B).

The infiltration of monocytes and macrophages into the aortic wall acts as a major source of proteolytic enzymes, including MMPs and cathepsins, which promote matrix degradation, thus impairing the integrity of the arterial wall and favoring aneurysm development $(20,26)$. MMPs such as MMP2 and MMP9 have been demonstrated as being critical for ECM remodeling and aneurysm 
A
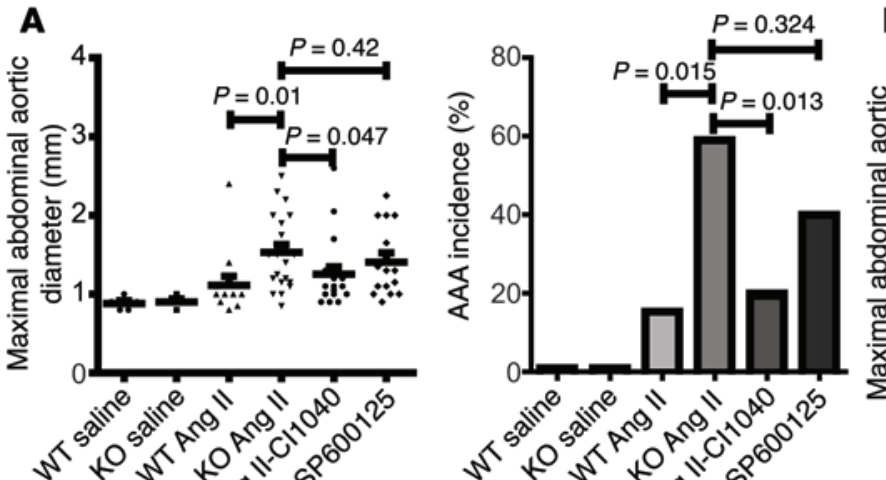

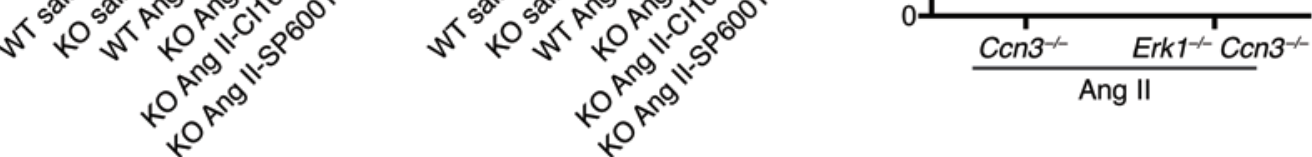
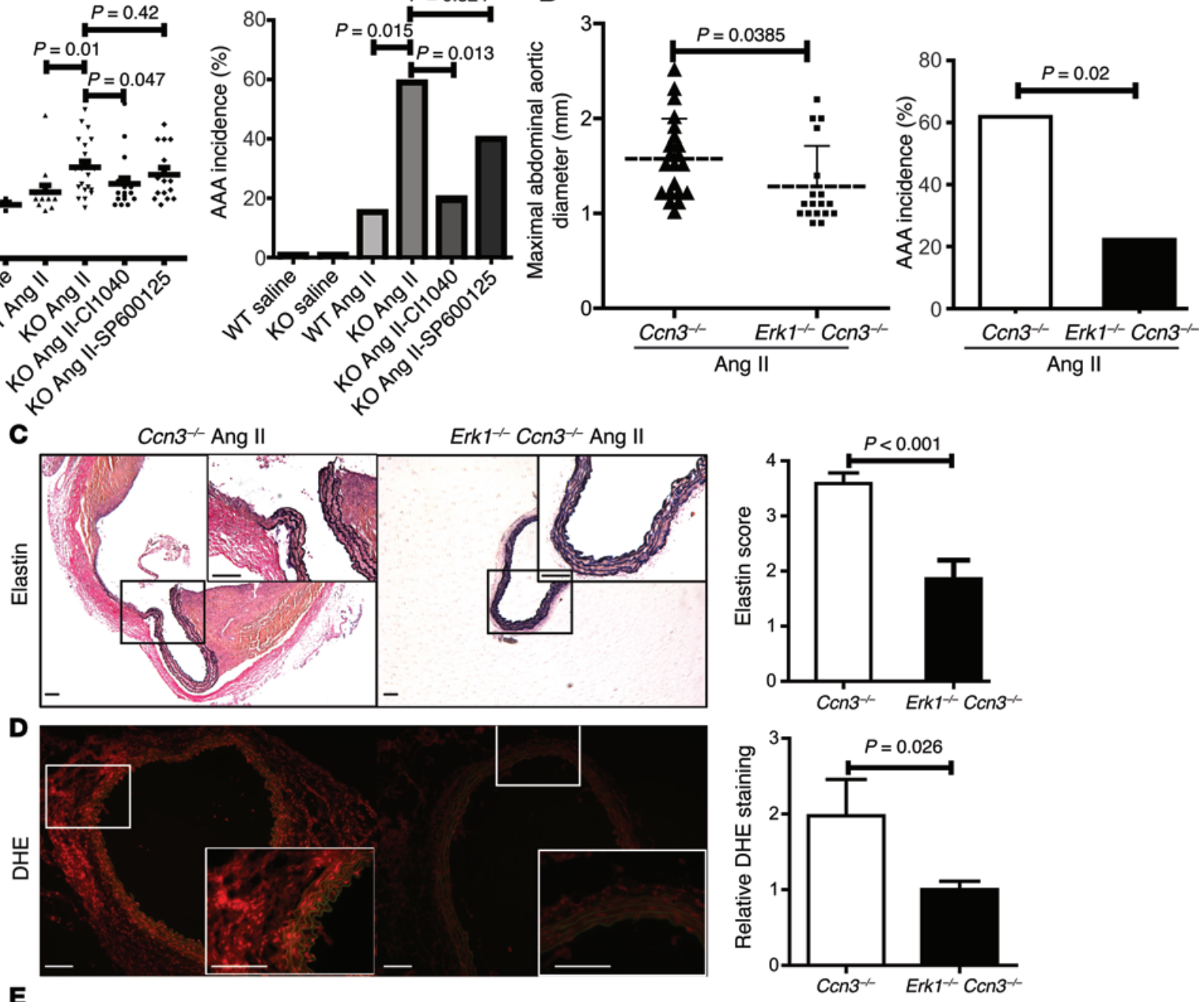

E
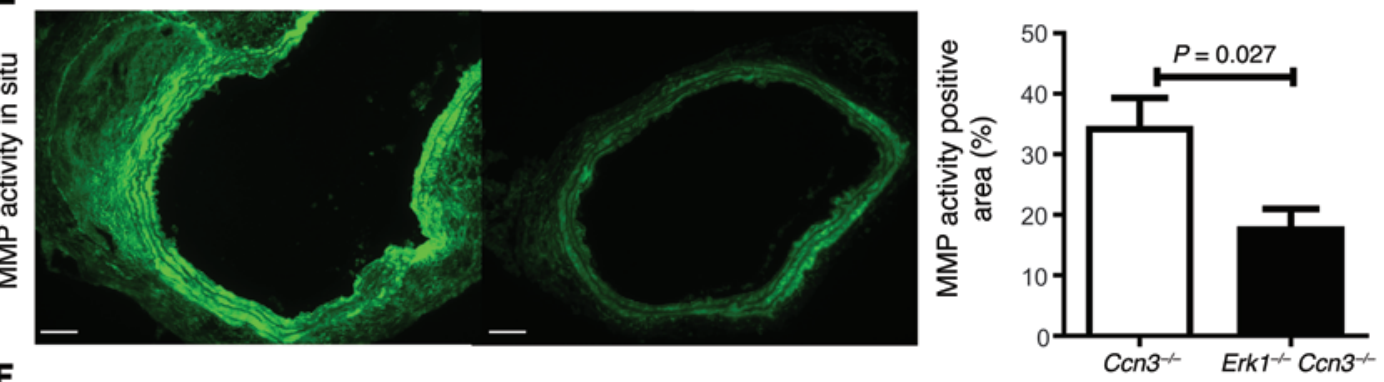

$\mathbf{F}$

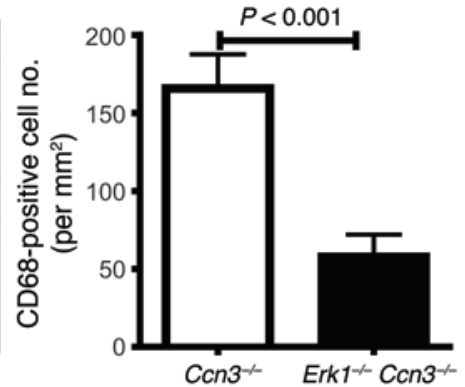


Figure 8. ERK1/2 pathway contributes to AAA formation in CCN3deficient mice. (A) Maximal abdominal aortic diameter (left panel) and AAA incidence (right panel) in response to pharmacological inhibition of ERK $1 / 2$ by $\mathrm{Cl}-1040$ and JNK $1 / 2$ by SP600125 in KO mice. $n=5$ for saline group; $n=13$ for WT with Ang II; $n=22$ for KO mice with Ang II; $n=20$ for KO mice plus Cl1040 group; $n=15$ for KO mice plus SP600125 group. (B) Analysis of genetic inhibition of Erk1 on maximal abdominal aortic diameter (left panel) and AAA incidence (right panel) using $[\mathrm{cn} 3 / E r k 1$ double-KO mice: Left panel, decrease of aortic expansion with Ang II treatment; right panel, AAA incidence, $\mathrm{CCn}^{-/-}, n=21 ; \mathrm{Cnn3}^{-/-} \mathrm{Erk1}^{-1-}, n=18$. Evaluation of elastin degradation ( $\left.\mathrm{Cnn}^{-/-}, n=17 ; \mathrm{CCn}^{-/-} \mathrm{ErkT}^{-/-}, n=7\right)(\mathrm{C})$, ROS produc-

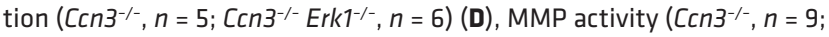
$\left.\mathrm{Ccn3}^{-1-} \mathrm{Erk1}^{-1-}, n=10\right)(\mathbf{E})$, and macrophage infiltration $(n=9)(\mathbf{F})$ in $\mathrm{Ccn3/}$ Erk1 compound-KO mice following Ang II infusion. Scale bars: $100 \mu \mathrm{m}$. For comparing AAA incidence, $\chi^{2}$ test was used; for all others, 2-way ANOVA followed by Bonferroni's post-hoc correction was used.

formation in experimental studies $(20,27)$. Given the severe elastin degradation observed in CCN3-deficient aortas (Figure 2G), we postulated that MMP expression was upregulated. As shown in Figure 4, A and B, and Supplemental Figure 8B, a marked increase of MMP2 and MMP9 was observed at both the protein and mRNA levels in the suprarenal abdominal aortas of CCN3-deficient mice following Ang II infusion. A similar augmentation of MMP2 was observed in the abdominal aortas of CCN3-deficient mice perfused with elastase (Supplemental Figure 6C). Consistent with the above findings, we observed a significant increase of MMP2 and MMP9 in the plasma of CCN3-deficient mice in response to Ang II infusion (Figure 4C). To determine whether altered MMP levels translate into proteolytic activity, we also evaluated MMP activity in aortic lysates from WT and CCN3-deficient mice by zymography. Consistent with the increase in MMP expression, considerably heightened MMP activity was observed in aortas of CCN3deficient mice compared with WT controls after Ang II infusion (Figure 4D). These results were further corroborated with in situ MMP activity assessment of aortic sections (Figure 4E).

In summary, these studies suggested that following Ang II infusion, the aortas of CCN3-deficient mice were characterized by an enhanced inflammatory state associated with elevated MMP activity.

Enhanced generation of ROS and loss of SMCs in CCN3-deficient mice in experimental AAA. Evidence from animal and human studies suggests that oxidative stress as a key mediator in the pathogenesis of AAA (28). ROS have been shown to play a key role in the regulation of MMPs and induction of SMC apoptosis (28). We therefore assessed oxidative stress. Abdominal aortic sections from Ang II- and saline-infused WT and CCN3-deficient mice were incubated with dihydroethidium (DHE). As expected, in saline-infused mice, both WT and CCN3-deficient aorta demonstrated minimal evidence of ROS. However, Ang II infusion for 7 days triggered a dramatic increase in markers of superoxide generation, with this effect being more pronounced in the absence of CCN3 (Figure 5A). Similar effects were observed following 28 days of Ang II infusion (Figure 5B and Supplemental Figure 9). The generation of ROS was also confirmed by the measurement of 4-hydroxynonenal (4-HNE), a major end product of lipid peroxidation. As shown in Supplemental Figure 10A, enhanced 4-HNE levels were observed in the aortas of CCN3-deficient mice compared with WT controls after Ang II infusion. There was also increased expression of p47Phox and cyclophilin A within the suprarenal abdominal aorta of CCN3-deficient mice (Supplemental Figure 10B). Both of these proteins have been implicated in generating ROS. Similar effects on 4-HNE are observed in the elastase perfusion model (Supplemental Figure 6D).

Healthy SMCs within the aortic media are important for normal vessel function. Aortic SMC apoptosis is a hallmark of human AAA (29). The CCN family of proteins has been shown to play a critical role in cell survival $(12,13)$. We therefore hypothesized that loss of CCN3 may promote exaggerated SMC apoptosis under the status of increased inflammation and elevated ROS seen in CCN3deficient mice after Ang II infusion. In the saline-infused animals, we observed minimal TUNEL staining in both control and CCN3deficient mice. After 7 days of Ang II infusion, marked TUNEL staining was observed in the aortic media of the CCN3-deficient mice (Figure 5C). In line with this observation, we found a marked difference in the staining pattern of SMC marker proteins between WT and CCN3-deficient mice following Ang II infusion. In WT controls, there was uniform staining for SMA in medial SMC and homogeneous layers of SMCs were separated by elastic laminae in an orderly pattern. In marked contrast, CCN3-deficient mice displayed a disrupted medial SMC layer with a dramatic paucity in SMA, suggesting a significant loss of SMC at 28 days after Ang II infusion (Figure 5D).

ROS are required for AAA formation in CCN3-deficient mice. Given the more profound ROS formation in the aortas of CCN3-deficient mice following Ang II infusion, we next sought to determine the causal role of ROS in the formation of AAA in CCN3-deficient mice. To address this question, pharmacological inhibition studies were employed using apocynin, an antioxidant that has been applied in a number of in vivo experimental studies for the purpose of inhibiting ROS production. Prior to AAA induction with Ang II infusion, 2 groups of male CCN3-deficient mice $(n=12$ per group) were administered water or apocynin $(100 \mathrm{mg} /$ $\mathrm{kg} / \mathrm{d}$ ) for 1 week. Next, all mice were subjected to Ang II infusion with continuous water or apocynin administration for 28 days. The efficacy of apocynin in limiting ROS was confirmed by DHE staining (Supplemental Figure 11A). As expected, the majority of CCN3-deficient mice $(66 \%)$ receiving water developed AAA. However, a strong reduction of AAA incidence was observed in CCN3-deficient mice receiving apocynin (25\%) (Supplemental Figure 11B). Furthermore, antioxidant application dramatically attenuated the major pathological features observed in CCN3deficient mice receiving water. As demonstrated in Supplemental Figure 11B, we observed a significant decrease in the maximal abdominal aortic diameter. Moreover, addition of apocynin to the drinking water preserved the vessel wall integrity evidenced by elastin staining (Supplemental Figure 11C), reduced the infiltration of macrophage by 66\% (Supplemental Figure 11D), and diminished MMP activity by 50\% (Supplemental Figure 11E). Finally, apocynin administration significantly mitigated Ang IIinduced ERK1/2 activation in CCN3-deficient mice (Supplemental Figure 11F). Collectively, these studies indicate ROS generation is a major contributor to AAA formation in CCN3-deficient mice.

$A A A$ formation in CCN3-deficient mice is intrinsic to the vessel wall. Our studies revealed an inhibitory role for the matrix-associated protein $\mathrm{CCN} 3$ in experimental AAA formation. However, 
A

C

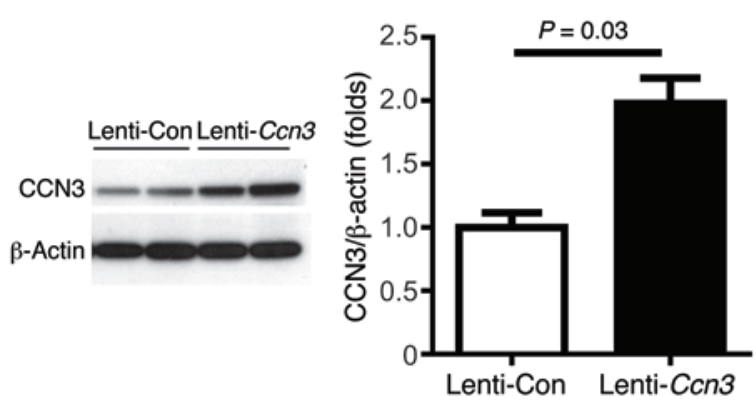

B
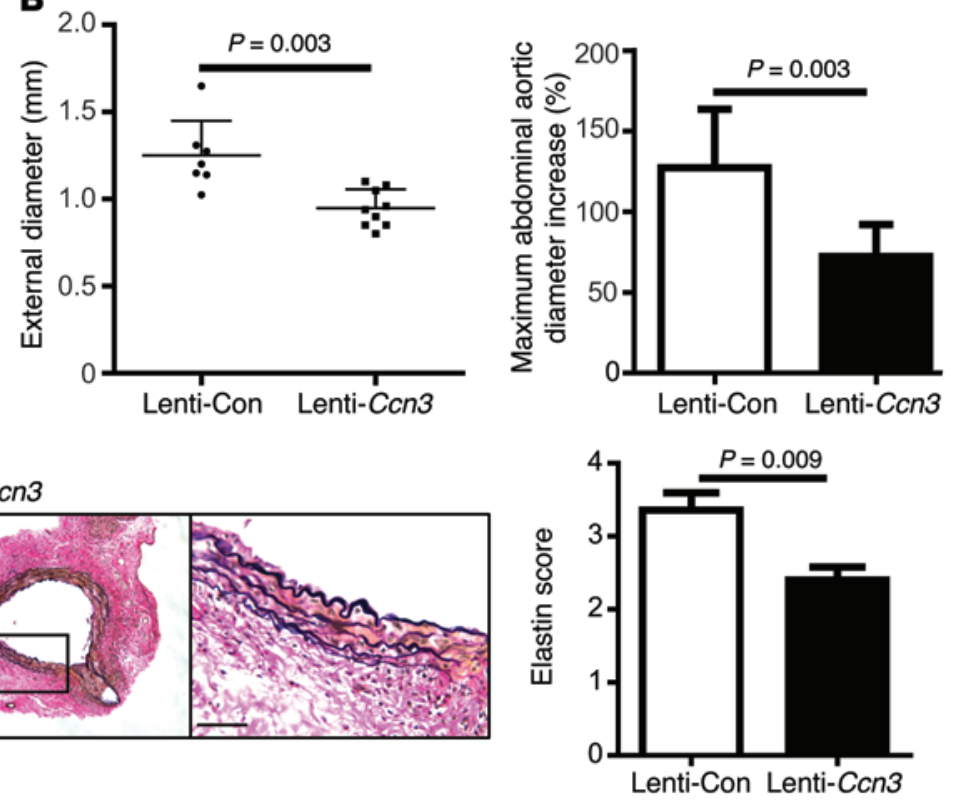

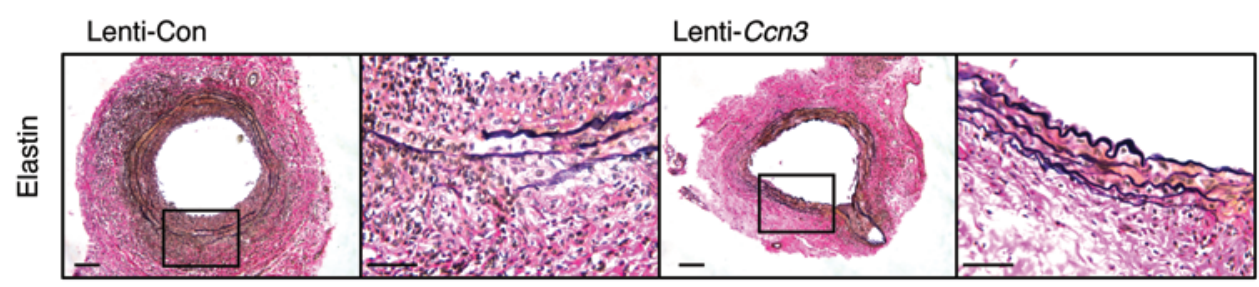

D

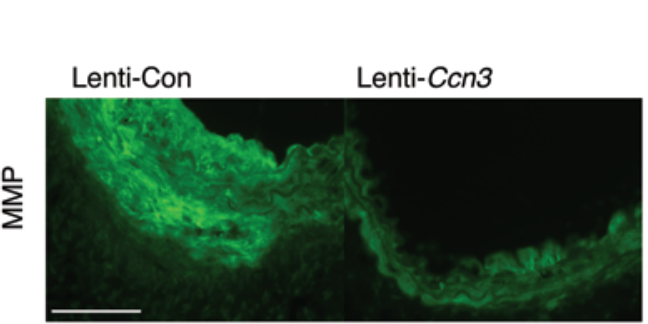

E

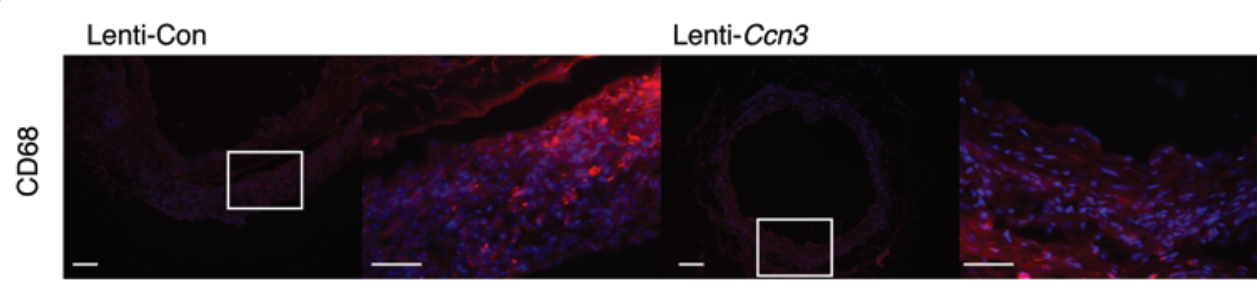

$\mathbf{F}$

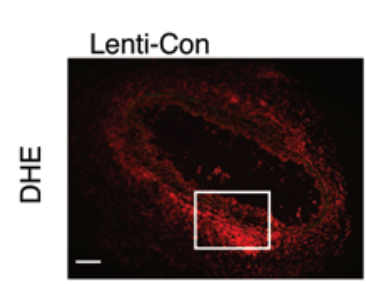

G
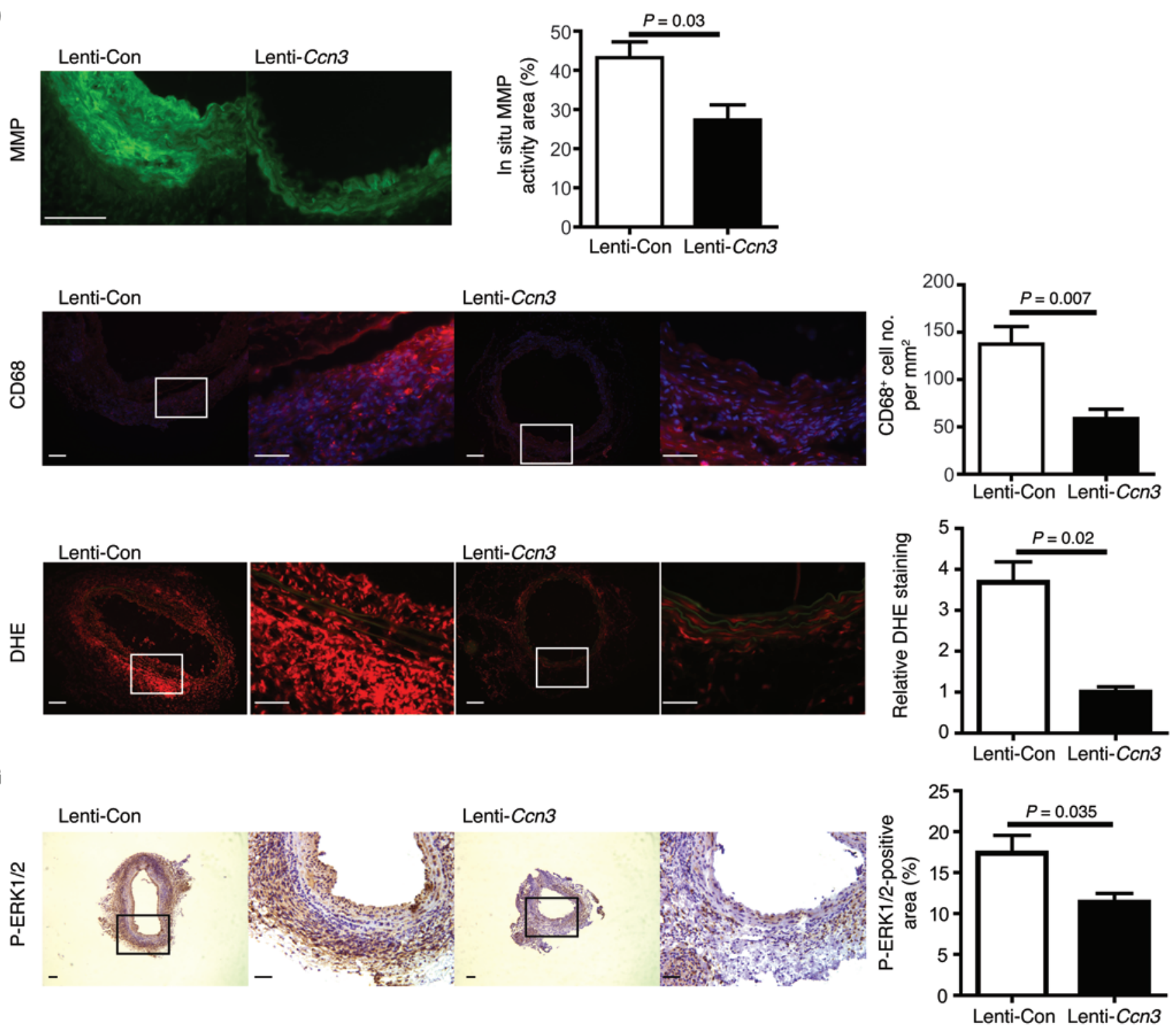
Figure 9. CCN3 overexpression inhibits elastase-induced AAA formation. (A) Confirmation of lentiviral CCN3 overexpression by Western blot. Left panel, representative Western blot showing CCN3 overexpression after 2 weeks of lentivirus injection; right panel, quantitation data for CCN3 Western blot $(n=5)$. (B) Infrarenal abdominal aortic dilatation 2 weeks after elastase perfusion in control (control lentivirus [Lenti-Con], $n=7$ ) versus CCN3 overexpression ( $C \mathrm{cn} 3$ lentivirus [Lenti- $C \mathrm{cn} 3], n=9$ ). Left panel, inhibition of external infrarenal aortic diameter; right panel, percentage of aortic diameter increase (Lenti-Con, $n=7$; Lenti-Ccn3, $n=9$ ). (C) Elastin staining by Verhoeff-van Gieson in abdominal aorta following elastase perfusion in control $(n=7)$ versus C cn3 overexpression aortae $(n=9)$. Assessment of MMP activity by in situ zymography $(n=5)(D)$; macrophage infiltration by immunostaining (Lenti-Con, $n=5$; Lenti-Ccn3, $n=9)(\mathbf{E})$; ROS production by DHE staining ( $n=6$ per group) (F); and ERK $1 / 2$ activation by immunohistochemical analysis (Lenti-Con, $n=7$; Lenti-Ccn3, $n=6$ ) (C). Scale bars: $100 \mu \mathrm{m}$. Two-way ANOVA followed by Bonferroni's post-hoc correction was used.

these observations were performed in CCN3 systemic KO mice an approach that precludes precise determination of the cellular contributor(s) within or outside of the vessel wall responsible for the AAA phenotype in vivo. To address this, we performed reciprocal BM transplantation (BMT) experiments. For these BMT studies, 12-week-old male mice were irradiated; this was followed by tail-vein injection of marrow from WT or CCN3-deficient mice. The experimental strategy is shown in Supplemental Figure 12. Transplanted mice were allowed 6 weeks of engraftment and were then subjected to Ang II infusion $(1,000 \mathrm{ng} / \mathrm{kg} / \mathrm{min})$ for an additional 28 days, at which time aortas were harvested and assessed for AAA development. We first assessed AAA formation using CCN3-deficient marrow as donor. These studies confirmed the development of severe AAA formation in 70\% of CCN3-deficient animals following 28 days of Ang II infusion, while CCN3deficient marrow reconstitution did not alter AAA development in WT mice. The mean maximal abdominal aortic diameter was $2.12 \pm 0.24 \mathrm{~mm}$ in the CCN3-deficient group $(n=11)$ receiving CCN3-deficient marrow, which is significantly bigger than that of the WT group transplanted with CCN3-deficient marrow (1.30 \pm $0.09 \mathrm{~mm}, n=11$, Figure $6 \mathrm{~A}$ ). These results strongly suggest that the AAA phenotype of CCN3-deficient mice is intrinsic to the vasculature. This notion is further substantiated by reverse BMT studies using WT as a donor. As shown in Figure 6, A and B, WT marrow did not alter the severity of AAA in CCN3-deficient mice. The maximal abdominal aortic diameter of CCN3-deficient mice receiving WT marrow was $1.9 \pm 0.29 \mathrm{~mm}(n=12)$, which was significantly larger than that of WT mice receiving WT marrow (1.22 $\pm 0.04 \mathrm{~mm}, n=14$, Figure 6A). In summary, these BMT studies support the hypothesis that vascular CCN3 plays a critical role in mediating the AAA phenotype.

The aortic wall is composed of 3 layers: the intima, composed of endothelial monolayer and underlying extracellular connective tissues; the media, consisting primarily of SMCs; and the outermost layer, the adventitia, containing fibroblasts and collagens, proteoglycans, and vasa-vasorum. Given the central importance of SMCs in the development of AAA coupled with the knowledge that CCN3 is highly expressed in SMCs of the aortic wall (Supplemental Figure 1), we hypothesized that alterations of CCN3 in SMCs may regulate key aspects of AAA pathology. We therefore next sought to determine the effects of SMC-derived
CCN3 on cellular ROS generation, apoptosis, and inflammatory response. Toward this end, we used primary mouse aortic SMCs isolated from control and CCN3-deficient mice. We first assessed whether loss of CCN3 affects ROS production. Murine vascular SMCs were serum deprived for 24 hours, which was followed by exposure to vehicle or Ang II for 24 hours. We then assessed ROS generation by DHE staining. As shown in Figure 6C, CCN3 deficiency resulted in augmented ROS production in SMCs, findings consistent with our in vivo observations in the vessel wall. Given these observations coupled with the profound impact of ROS on SMC apoptosis, we next tested the effect of CCN3 deficiency on SMC survival. Here, SMCs received vehicle or Ang II and the degree of apoptosis was analyzed by a TUNEL assay. As shown in Figure 6D, Ang II administration resulted in apoptosis of WT SMCs; however, in the absence of CCN3, Ang II administration caused exacerbated apoptosis of SMCs. The findings were consistent with enhanced apoptosis in SMCs observed in the aortic wall after Ang II administration (Figure 5C). In addition, we analyzed mRNA levels of several inflammatory markers (Mcp1, Icam1, Il6, and Vcam1) from vascular SMCs receiving Ang II. qPCR results indicated that loss of CCN3 resulted in enhanced Mcp1, Icam1, Il6, and Vcam 1 expression (Figure 6E). These data suggest a proinflammatory state in the CCN3-deficient SMCs. Conversely, adenoviral-mediated overexpression of CCN3 in rat aortic SMCs (RASMCs) significantly reduced ROS generation as well as Mcp1, Icam1, Il6, and Vcam1 expression (Supplemental Figure 13, A and B). Taken together, our results indicate that alterations of CCN3 in vascular SMCs cause multiple functional changes, supporting the notion that loss of vascular SMC-derived CCN3 plays an important role in AAA formation.

Pharmacological and genetic inhibition of the ERK1/2 pathway mitigates Ang II-induced AAA formation in CCN3-deficient mice. Mechanistic studies over the last several decades have focused on key signaling pathways underlying AAA initiation and progression, including MAPKs, NF-KB, TGFB, STAT, and mTOR (30-37). These pathways have been shown to regulate vascular inflammation, ROS elaboration, cell survival, and vessel wall integrity. In an effort to glean mechanistic insights into the underlying cause of AAA pathology in CCN3-deficient mice, we first surveyed several MAPK and TGFB signaling pathways. Protein lysates from Ang II-infused abdominal aortas as well as aortic sections were subjected to Western blot and immunohistochemistry analysis. As shown in Supplemental Figure 14, these studies indicated that CCN3 deficiency does not affect Smad2 phosphorylation (indicative of the TGFB pathway), the JNK pathway, the NF-kB pathway, or p38 kinase. However, we observed a profound activation of ERK1/2 signaling in CCN3-deficient aortas, as evidenced by marked enhancement of ERK1/2 phosphorylation at both 7 days and 4 weeks after Ang II infusion by both immunohistochemical and Western blot analyses (Figure 7, A-C). In addition, a significantly heightened activation of MEK1/2 (the upstream activator of ERK1/2) was observed in CCN3-deficient aortas (Figure 7C). Furthermore, immunohistochemistry studies revealed a more robust ERK1/2 phosphorylation in the media of CCN3-deficient aortas (Supplemental Figure 15). A similar enhanced activation of ERK1/2 was observed in CCN3-deficient aortas following elastase perfusion (Supplemental Figure 16). Finally, Western 
blot analysis was performed to assess whether CCN3 deficiency affects ERK1/2 signaling in SMCs. Following Ang II administration, we observed elevated ERK1/2 activation and its upstream regulator MEK1/2 in CCN3-deficient SMCs compared with WT SMCs (Figure 7D). Conversely, overexpression of CCN3 in RASMC significantly inhibited ERK1/2 and MEK1/2 phosphorylation (Supplemental Figure 13C). These observations prompted us to hypothesize that ERK1/2 activation may govern the effects of AAA formation in CCN3-deficient mice.

To determine whether ERK1/2 activation plays a causal role in AAA observed in CCN3-deficient mice, we undertook both pharmacological and genetic inhibition approaches. Our first approach was to utilize CI-1040, an inhibitor of MEK that has been widely used in animal studies. CCN3-deficient mice $(n=12)$ were pretreated with vehicle or CI-1040 (100 mg/ $\mathrm{kg} / \mathrm{d})$ by gavage for 3 days. Thereafter, all mice were infused with Ang II for 28 days. During the ensuing Ang II infusion period, the 2 groups of mice continually received daily oral gavage of vehicle or CI-1040. In light of the reported inhibitory effects on AAA development in rodents (30), we performed a parallel experiment using a JNK pathway inhibitor, SP600125. Western blotting was performed to verify the successful inhibition of ERK1/2 activation (Supplemental Figure 17). Upon completion of Ang II infusion, blockade of ERK1/2 signaling mitigated the CCN3-dependent Ang II induction of AAA formation and AAA incidence, as demonstrated by diminished abdominal aortic dilation (Figure 8A). Interestingly, JNK inhibition caused a mild reduction of aorta expansion, but the effects were not significant (Figure 8A), indicating JNK activation does not play a major role in the AAA pathology of CCN3-deficient mice.

In order to further corroborate our findings garnered from these pharmacological inhibition studies, we also carried out studies with genetic inactivation of ERK1 in CCN3-deficient mice. Erk1-KO mice are viable, whereas Erk2 deficiency is embryonically lethal (38), thus precluding us from including Erk2-null mice in this study. We backcrossed CCN3-deficient mice onto Erk1-KO mice (both on C57BL/6 background). Subsequently, both CCN3-deficient and CCN3/ERK1 compound-KO mice underwent 28 days of Ang II infusion and AAA phenotype was assessed. In marked agreement with the results from the pharmacological inhibition experiments, genetic inactivation of ERK1 dramatically reduced AAA incidence and severity, as assessed by maximal aortic diameter and elastin breakdown in the vessel wall (Figure 8, B and C). Moreover, loss of ERK1 signaling strongly reversed ROS production, MMP activity, and macrophage infiltration (Figure 8, C-F). Collectively, these findings indicate that hallmark features of AAA phenotype in CCN3-deficient aortas are ERK1/2 dependent.

Virus-mediated overexpression of CCN3 is sufficient to prevent AAA development in a murine AAA model. As presented above, data derived from our loss-of-function studies strongly implicate CCN3 as playing an important role in the pathogenesis of aortic aneurysm. An important question that remains is whether sustained CCN3 expression in animals confers salutary effects on the development of AAA. We first tested the effects of overexpression in the elastase perfusion model. To achieve sustained CCN3 expression in vivo, we adopted a lentivirus-mediated overexpression approach that has been successfully employed by a number of groups (3941). We performed tail-vein injection of either control lentivirus or
lenti-Ccn3 virus $\left(1.0 \times 10^{8} \mathrm{IFU}\right.$ [infectious units]/mouse). One day prior to lentivirus injection, mice were subjected to elastase perfusion as described in Methods. Mice were then injected with lentivirus on the following day. Two weeks later, AAA extent and severity were evaluated by ultrasound, morphology, and biochemistry. CCN3 overexpression in the vessel was verified by Western blot (Figure 9A). Using this approach, systemic delivery of lenti-CCN3 resulted in 2.0-fold CCN3 overexpression in the aorta. Strikingly, sustained CCN3 expression in vivo strongly blunted the progression of AAA induced by elastase perfusion. Following 2 weeks of elastase perfusion, the maximum aortic diameter increased by $127.3 \% \pm 13.73 \%(n=7)$ in the control virus group compared with baseline. However, the extent of aortic dilation was significantly lower in the CCN3 overexpression group $(72.32 \% \pm 6.59 \%, n=9$, $P=0.003$, Figure 9B). The severity of elastin destruction was also much less pronounced in the CCN3-overexpressing mice (Figure 9C), consistent with the markedly reduced MMP activity in these mice (Figure 9D). We then analyzed macrophage infiltration and ROS production. As shown in Figure 9, mice with CCN3 overexpression exhibited a significant reduction in macrophage infiltration (Figure 9E) and ROS generation (Figure 9F). Finally, to complement loss-of-function studies where heightened ERK1/2 signaling in CCN3-deficient aorta was observed, we assessed for ERK1/2 phosphorylation in the setting of CCN3 overexpression. As evidenced by immunohistochemistry data, sustained CCN3 expression markedly diminished ERK1/2 activation (Figure 9G), supporting the notion that the CCN3 suppression of AAA is, at least in part, through a mechanism involving ERK1/2 signaling inhibition. These results suggest that lentivirus-mediated CCN3 overexpression is sufficient to prevent AAA progression in the elastase perfusion model. To determine whether a similar inhibitory effect could be achieved in the Ang II model, we carried out studies using adenovirus-mediated overexpression, an approach that has been successfully used during Ang II infusion into Apoe-null mice (42). Control and CCN3 adenovirus were administered locally to the suprarenal aorta of Apoe-null mice, followed by Ang II infusion the next day. Mice were sacrificed at day 14 after Ang II infusion and abdominal aortic dilation evaluated. As shown in Supplemental Figure 18, adenoviral overexpression of CCN3 significantly ameliorated Ang II-mediated abdominal aortic expansion, further corroborating that $\mathrm{CCN} 3$ is able to prevent AAA progression.

\section{Discussion}

Previous studies demonstrate that CCN proteins control key cellular processes, such as apoptosis, senescence, mitosis, adhesion, ECM production, differentiation, proliferation, growth arrest, and migration $(13,43)$. Given these roles, it is not surprising that aberrant expression of CCN proteins has been implicated in the pathobiology of a wide array of diseases, including cancer, diabetic nephropathy, and systemic sclerosis (13). With regard to the cardiovascular system, a causal role for the CCN family of proteins in arterial pathology has not been previously demonstrated. Our studies reported here thus provide what we believe to be novel evidence implicating CCN3 in the pathobiology of AAA. This is supported by a number of observations. First, we show that CCN3 expression is attenuated in both human and rodent AAA tissues. Next, genetic ablation of CCN3 exacerbates vascular dilation and 
elastolysis in rodent AAA models. Further, our results demonstrate that CCN3 is a negative regulator of multiple key pathological processes involved in aneurysm formation, including oxidative stress, MMP elaboration, SMC apoptosis, and vascular inflammation. BMT experiments, coupled with ex vivo cell culture studies, support the notion that SMC-derived CCN3 may be an important regulator of AAA. Mechanistic studies reveal a role for ERK1/2 activation in the pathogenesis of AAA in CCN3-deficient mice, results corroborated by both pharmacological and genetic inhibition studies. Finally, we show that systemic overexpression of CCN3 prevents AAA formation.

The results of this study advance our understanding of CCN proteins in vascular biology and offer insights into other vascular abnormalities. Recent studies have revealed the roles of CCN proteins in angiogenesis, effects attributed to their interactions with integrins (44-46). Alterations of CCN1 and CCN2 expression were observed in atherosclerotic lesions, suggesting a potential role of these 2 factors in atherosclerosis $(47,48)$. CCN2 has also been previously reported to be upregulated in aneurysmal and dissected arteries (49). At the cellular level, several CCN members (CCN1, CCN2, CCN3, CCN4) have been shown to regulate the synthetic phenotype of SMCs $(17,50-53)$. Our recent study suggests CCN3 is a flow-inducible factor and a negative regulator of endothelial inflammation (16). In our current study, we provide evidence to suggest an important role of CCN3 in regulating AAA disease through ROS- and ERK1/2-dependent mechanisms, thus establishing the first example, to our knowledge, of the involvement of the $\mathrm{CCN}$ family in vascular disease. By virtue of the versatile ability of matricellular protein CCN3 to impart on multiple aspects of vessel pathology, including vascular remodeling, inflammation, cell survival, and ROS production, one can postulate that CCN3 could modulate an essential stage common to many inflammatory diseases such as vasculitis and atherosclerosis. Previous studies on AAA commonly employed dyslipidemic mice infused with Ang II. CCN3-deficient mice developed AAA in response to Ang II on a nonhyperlipidemic background, suggesting that the Ccn3-mutant mice may serve as a model for investigating AAA independent of lipid levels.

Given that vascular wall integrity is critical to maintaining its function, we further assessed the effect of CCN3 deficiency on vascular structure. Histology and electron microscopy studies revealed no obvious abnormalities in the aortic wall of Ccn3-deficient mice, suggesting CCN3 does not affect the formation of elastic fibers or affect vascular stability. These findings are in line with the current understanding of matricellular proteins, which play a minimal role in vascular structure, but are primarily involved in the regulation of cellular functions (13).

Next, we investigated the mechanistic basis for the protective role of CCN3 in AAA formation by examining multiple signaling pathways known to be important for AAA pathogenesis. These signaling pathways are stimulated with Ang II and regulate inflammatory and apoptotic targets. Our genetic and pharmacological studies suggest that the ERK1/2 pathway is a major regulator of Ang II-induced AAA formation in CCN3-deficient mice. However, we are aware that ERK1/2 might not be the sole mechanism by which $\mathrm{CCN} 3$ alters the prooxidative, proinflammatory, and proapoptotic response in the observed aortic aneurysm phenotype of CCN3- deficient mice. Indeed, there are an abundance of signaling pathways, including Rho/ROCK, p53, PI3K/Akt, NOTCH, AMPK, and STAT, known to be critical in AAA pathology that were not investigated in the current study, but that may also play an important role in AAA pathology $(25,33,54-60)$. For example, SMC-derived AMPK- $\alpha 2$ has been shown to be critical for nicotine- and Ang IImediated AAA progression and abrogation of AMPK- $\alpha 2$ blunts AAA formation (60). KO of Akt2 also exacerbates AAA formation (61). Thus, the potential role of CCN3 in altering these pathways in AAA formation requires further investigation.

Another important question of this study relates to the cellular contributor or contributors responsible for the AAA phenotype. Considering that both vascular resident cells and recruited myeloid-derived inflammatory cells contribute to the vascular inflammatory response and matrix degradation (2 key drivers in the formation of AAA), we performed reciprocal BMT experiments. Although our reciprocal BMT experiments point to the importance of vessel-derived CCN3 in conferring protection from AAA formation, this approach does not distinguish between the vascular wall and other somatic tissues. This limitation also applies to our systemic delivery of CCN3 using a viral approach in the overexpression studies, in which the hepatic expression of CCN3 is likely to increase, which could potentially affect the overall physiology of the animals. Furthermore, there are 3 cell types within the vessel wall: ECs, vascular SMCs, and adventitial fibroblasts. Given their central importance in vascular structure and function, SMCs have been the most studied cell type for gaining mechanistic insights involved in AAA formation. We therefore assessed the effects of alteration of CCN3 levels on SMC function. Our in vitro studies using cultured SMCs indeed support the important role of CCN3 in regulating inflammation, ROS, and ERK1/2 signaling (Figure 4 and Supplemental Figure 13). However, these results do not provide definitive proof of the importance of SMC. Additionally, it is noteworthy CCN3 is expressed in the hematopoietic compartment $(62,63)$. Therefore, future studies using tissue-specific Ccn3-KO mice are warranted to definitively determine the precise cellular contributor or contributors in AAA pathology.

Our studies suggest clinical relevance of $\mathrm{CCN} 3$ to AAA. We found a marked reduction of CCN3 in human AAA tissues in comparison with age- and sex-matched nonaneurysmal abdominal aorta, implicating the relevance of alterations of CCN3 expression in human aneurysm formation. To characterize the role of CCN3 in the pathogenesis of AAA, the current study utilized 2 established rodent models to examine the effects of germline deficiency of Ccn3 on AAA formation, both of which recapitulate human AAA pathology. The Ang II infusion model is known to elicit AAAs with some features of human AAA, including dramatic aortic dilatation with aneurysm formation, aortic media destruction, and inflammatory cell recruitment and infiltration as well as ROS and MMP expression (22). The AAA phenotype in CCN3-deficient mice remarkably resembles the above-mentioned features in human AAA. However, one limitation to this model is that AAA developed in the suprarenal aorta, whereas human AAA usually occurs in the infrarenal aorta. To circumvent this issue and to more closely mirror the human AAA, we adopted the elastase perfusion model. The advantage of this model is that an aneurysm is formed in the infrarenal aorta. The finding that CCN3 deficiency promotes AAA in 
this second model underscores the importance of CCN3 in maintaining normal vascular health. The pleiotropic beneficial effects that CCN3 exerts to curtail AAA pathology (vascular inflammation, SMC loss, MMP elaboration, and ROS production) suggest that therapeutic upregulation of CCN3 could be a viable strategy to limit AAA growth. In support of this notion, our studies show that systemic increase of CCN3 levels in mice mitigates the progression of AAA. These findings thus provide proof of concept for nonsurgical therapies to halt aneurysm progression.

In summary, we identify matricellular protein $\mathrm{CCN} 3$ as a regulator of AAA formation. Our studies point to an important contribution of ERK1/2 in driving AAA pathology in the context of CCN3 deficiency. Moreover, our studies suggest that augmenting CCN3 levels may have therapeutic benefits in controlling aortic expansion. Future work to gain in-depth understanding of CCN3 action may facilitate the development of a novel medical strategy against this vascular disease.

\section{Methods}

Mice. Ccn3-KO mice were generated as previously described (17). Erk1KO mice were provided by Gary Landreth (Case Western Reserve University) (64). WT C57BL/6 and Apoe-null mice were purchased from the Jackson Laboratory and maintained in our animal facility. All control and mutant mice used in this study were on a pure C57BL/6 background. Mice were housed under standard light conditions (12-hour light/12-hour dark cycle) and allowed ad libitum access to standard rodent chow and water.

Ang II infusion model. To induce AAA formation in mice, male mice at the age of 10 to 12 weeks were infused with Ang II as previously described (65). Alzet model 1007D, 1002, or 1004 osmotic minipumps (DURECT Corp.) implanted subcutaneously were used to deliver Ang II (1000 ng/kg/min, Sigma-Aldrich) or vehicle (normal saline) for 7, 14, and 28 days, depending on the experimental design described in Results. Mice were anesthetized with ketamine (1.5 $\mathrm{mg} / \mathrm{kg})$ and xylazine $(0.3 \mathrm{mg} / \mathrm{kg})$, which was followed by minipump implantation. To relieve pain from surgery, postoperative analgesia was administered using intraperitoneal injection of buprenorphine (0.05-0.10 mg/kg administered every 8 to 12 hours) on the day of surgery and on postoperative days 1 to 3 . To assess the severity of AAA formation, the methods described by Daugherty et al. were adopted (24). AAA is classified as follows: type I, dilated lumen in the suprarenal aorta with no thrombus; type II, remodeled tissue in the suprarenal region that frequently contains thrombus; type III, a pronounced bulbous form of type II that contains thrombus; and type IV, multiple aneurysms containing thrombus, some overlapping, in the suprarenal area of the aorta (Supplemental Figure 19). At the completion of Ang II infusion, mice were perfused with normal saline followed by $4 \%$ paraformaldehyde at $100 \mathrm{mmHg}$ for 8 minutes. The aorta was exposed under a dissecting microscope, periadventitial tissues were carefully removed from the aortic wall, and a caliper was used to measure the external diameter of suprarenal abdominal aorta. To determine AAA incidence, an aneurysm was defined by a $50 \%$ or greater increase in the external diameter of the suprarenal aorta in comparison with aortas from saline-infused WT mice.

Elastase-induced AAA model. Elastase perfusion in mice was performed as previously described (20). Briefly, 8- to 12-week-old male mice were anesthetized with ketamine and xylazine, the infrarenal aorta was isolated, and temporary ligatures were placed around proximal and distal portions of the infrarenal aorta using 6-0 suture. Porcine pancreatic elastase (Sigma-Aldrich, $0.4 \mathrm{U} / \mathrm{ml}$ ) was used to perfuse the infrarenal aorta through a syringe pump for 5 minutes at a pressure of $100 \mathrm{mmHg}$. Echocardiography was performed to measure infrarenal aorta diameter 1 day prior to and 7 and 14 days after perfusion by using the Vevo 770 High Resolution Imaging System (Visual Sonics) and the RMV-707B 30- MHz probe.

$B M T$. Twelve-week-old control and CCN3-deficient mice were lethally irradiated (11 Gy). To obtain donor BM cells, femurs and tibias from both WT and CCN3-deficient mice were flushed with DPBS containing $2 \%$ FBS (Atlantics). A total of $2 \times 10^{6}$ of unfractionated BM cells were injected into the recipient mice through the lateral tail vein. Following 6 weeks of engraftment, mice were subjected to Ang II or saline infusion for 28 days as described above.

Apoptosis assay. Confluent primary SMCs grown in 24-well plates were serum deprived for 24 hours, after which cells were treated with vehicle or Ang II $(1 \mu \mathrm{M})$ for 24 hours. Experiments were performed 4 times using 4 separate SMC isolations, and for each experiment, treatment with Ang II or saline was carried out in triplicate. Aortic sections were deparaffinized and used for cell apoptosis assessment. For both in vitro and in vivo experiments, TUNEL staining was carried out to detect apoptotic cells using In Situ Cell Death Detection Kit according to the manufacturer's instructions (Roche Diagnostics Co.). DAPI counterstaining was performed to reveal total nuclei on each culture well or aorta sections using VECTASHIELD mounting medium (Vector Laboratories). For aortic sections, the number of TUNEL-positive nuclei per section was normalized to total nuclei using Image-Pro Plus software. Results were presented as mean \pm SEM of the percentage of apoptotic cells.

ROS generation assay. To evaluate ROS production in primary aortic SMCs, following 24 hours of Ang II treatment, cells were incubated with DHE ( $5 \mu \mathrm{M}$, Molecular Probes) for 30 minutes. Aortas were perfused with ice-cold PBS ( $\mathrm{pH} 7.4$ ) at $100 \mathrm{mmHg}$ for 5 minutes at $4^{\circ} \mathrm{C}$. The abdominal aorta was dissected and embedded in OCT (TissueTek, Miles Inc.). DHE ( $5 \mu \mathrm{M})$ was topically applied to the freshly cut frozen aortic sections $(10 \mu \mathrm{m})$ and incubated at $37^{\circ} \mathrm{C}$ for 30 minutes. The presence of ROS is shown as red. DHE fluorescence images were acquired with an Olympus microscope using $510 \mathrm{~nm}$ excitation/580 $\mathrm{nm}$ emission filters at the same photomultiplier tube voltage, gain, and offset. Images were converted to gray scale, and the integrated density per image area of interest was measured using NIH Image (http:// imagej.nih.gov/ij/).

Cell, tissue lysis, and Western blot. Cells were treated as described and then harvested for total protein by RIPA buffer supplemented with protease inhibitor tablet (Roche). Snap-frozen aorta tissues were extracted using a Total Protein Extraction Kit with protease and phosphatase inhibitor from Millipore. Tissues were homogenized using a pellet pestle motor (Kimble-Kontes) according to the protocol in the protein extraction kit. Protein concentrations were determined by BCA kit (Pierce). Equal amounts of the resultant protein were subjected to Western blot analyses using the indicated antibodies. Membranes were reprobed with antibodies against $\beta$-actin (Santa Cruz Biotechnology Inc.) to confirm equal loading. For overexpression studies in RASMC, cells were infected with control virus (Ad-GFP) or Ad-CCN3 for 48 hours, followed by treatment with Ang II $(1 \mu \mathrm{M})$ for 30 minutes, and then harvested for total pro- 
tein. The resultant protein was subjected to Western blot analyses using the indicated antibodies. CCN3 proteins in the conditioned medium were concentrated using heparin sepharose beads (Amersham) as described by Chevalier et al. (66). Briefly, supernatants were incubated overnight with heparin and then washed 4 times in PBS containing protease inhibitors. Bound CCN3 was dissociated using 2-mercaptoethanol in Laemmli buffer, boiled for 10 minutes, and then centrifuged. The supernatant was collected for Western blot assay of the free protein. Heparin sepharose-concentrated samples and cellular extracts were subjected to Western blot analyses using CCN3 (K19M) antibody (66). Other primary antibodies are listed in Supplemental Table 1.

MMP activity measurement in tissue lysate and in situ. MMP2 and MMP9 activities in abdominal aorta tissue homogenate were determined by zymography. Equal amounts of nonreduced aorta lysate were run on Novex 10\% Zymogram (gelatin) Protein Gels (Invitrogen). After electrophoresis, gels were renatured with Novex Zymogram Renaturing Buffer for 30 minutes at room temperature. The gels were then put in developing buffer and incubated overnight at $37^{\circ} \mathrm{C}$. Finally SimplyBlue Safestain (Invitrogen) was applied to visualize protease activity. In situ MMP zymography was performed on freshly cut OCT-embedded aorta sections. Fluorescein-conjugated gelatin substrate DQ gelatin (Invitrogen) was prepared according to the manufacturer's instructions. The substrates were then applied to sections and allowed to incubate at $37^{\circ} \mathrm{C}$ for 24 hours, after which green fluorescence was examined using a fluorescent microscope. Negative controls were performed on parallel sections in the presence of $5 \mathrm{mM}$ EDTA. Pictures were taken and fluorescence intensity calculated with Image $(67)$. The results were expressed as percentage of fluorescence area over each cross section.

Lentivirus- and adenovirus-mediated overexpression. C57BL/6 male mice ( 8 to 10 weeks old) underwent elastase perfusion surgery as described above. One day prior to surgery, mice underwent injection of lentivirus that harbored mouse Ccn3 cDNA (lentivirus generated by Welgen) or control virus. To achieve sustained expression of CCN3 in mice, control or Ccn3 lentivirus $\left(1.0 \times 10^{8} \mathrm{IFU}\right)$ was injected into elastase-treated $\mathrm{C} 57 \mathrm{BL} / 6$ mice via tail vein. The infrarenal aorta diameter was measured at $-1,7$, and 14 days after elastase perfusion by echocardiography. Fourteen days following elastase perfusion, mice were sacrificed and external infrarenal aorta diameters were measured. The mice were perfused with ice-cold PBS, and the infrarenal aorta was segmented in half and embedded in paraffin and OCT medium, respectively. Paraffin sections were used for H\&E and elastin staining as well as immunohistochemical analysis. Freshly cut OCT sections were used for ROS generation assessment by DHE staining. Thoracic portions of aortas were snap-frozen for total protein extraction and assessed for $\mathrm{CCN} 3$ protein expression. To assess the effect of perivascular Ccn3 overexpression in the Apoe ${ }^{-/-}$mouse AAA model, control or Ccn 3 adenovirus $\left(1 \times 10^{10} \mathrm{PFU}\right.$, generated by Welgen) was injected directly into the adventitial space of suprarenal abdominal aorta, which was followed by Ang II pump implantation on the same day. The suprarenal aorta diameter was measured at -14 days after Ang II infusion.

In vivo ERK1/2 inhibition. Two approaches were undertaken to address the importance of Erk1/2 activation in AAA formation in CCN3-deficient aorta. To pharmacologically inhibit ERK1/2 activation, CI-1040 (Selleck Chemicals), a well-studied MEK inhibitor, was used. CI-1040 was suspended in an 8:1:1 PBS:ethanol:cremophore solution. CCN3-deficient mice ( $n=12$ per group) were pretreated with vehicle or CI-1040 (100 mg/kg/d) by gavage for 3 days. A parallel experiment was conducted using JNK inhibitor (SP600125, $50 \mathrm{mg}$ / $\mathrm{kg} / \mathrm{d}$, Sigma-Aldrich) in a separate cohort of CCN3-deficient mice. These pretreated mice were then subjected to Ang II infusion for 28 days, after which AAA formation was assessed. To genetically inhibit ERK1/2 activation, CCN3-deficient mice were crossed with Erk1-null mice to generate $C c n 3 / E r k 1$ compound-KO mice. The resultant mice were treated with saline or Ang II as described above.

ROS inhibition studies. To inhibit the formation of ROS in vivo, apocynin (Acros Organics) was used. Two groups of Ccn3-KO mice ( $n=12$ in each group) were included in this study. One group of mice received water supplemented with apocynin; the control group received a similar amount of water. All mice were allowed ad libitum access to standard rodent chow. Mice were pretreated with apocy$\operatorname{nin}(100 \mathrm{mg} / \mathrm{kg} / \mathrm{d})$ for 7 days prior to Ang II infusion, after which an osmotic minipump was implanted subcutaneously to deliver Ang II $(1000 \mathrm{ng} / \mathrm{kg} / \mathrm{min})$ for 28 days with concurrent apocynin treatment. At the end of the study, mice were sacrificed and AAA severity assessed as above; tissue was harvested for biochemical and morphological analysis.

Histology and immunohistochemistry. These studies were performed according to standard procedures. Please refer to Supplemental Methods for details. Elastin fragmentation was graded according to the criteria illustrated in Supplemental Figure 20. Primary antibodies are listed in Supplemental Table 2.

Additional experimental procedures are presented in Supplemental Methods.

Statistics. Data are expressed as mean \pm SEM. To compare the incidence of AAA formation between 2 groups, $\chi^{2}$ test and 2-tailed Fisher's exact probability test were used. For comparison of the differences in CCN3 mRNA levels between non-AAA and AAA tissues, Mann-Whitney $U$ test was used. To compare the differences within 3 or more levels within 1 variable, 1-way ANOVA was used. For comparison of the effects of 2 variables, 2-way ANOVA followed by Bonferroni's posthoc correction was used. $P \leq 0.05$ was considered significant.

Study approval. For human samples used in RNA analysis, AAA tissue was collected from patients undergoing AAA repair, as previously described (19). Control abdominal aortic specimens were matched to cases using sex, age, and ethnicity, as described (19). All aortic specimens were obtained by protocols approved by the Institutional Review Board of Wayne State University. All tissues were collected in RNAlater (Ambion), followed by RNA extraction. A separate set of aortic tissues used in immunohistochemistry studies was obtained by protocols approved by the Human Investigation Review Committee at Brigham and Women's Hospital. Samples were immediately immersed in saline, embedded in OCT, and stored at $-80^{\circ} \mathrm{C}(68)$. These sections were used in evaluating $\mathrm{CCN} 3$ expression in human aorta samples from non-AAA control and AAA patients. All animal protocols were approved by the Institutional Animal Care and Use Committee at Case Western Reserve University, which is certified by the American Association of Accreditation for Laboratory Animal Care.

\section{Author contributions}

$\mathrm{CZ}$ and ZL conceived and designed the research studies. CZ, DVDV, HS, RZ, YQ, and DAP performed experiments and/or 
interpreted data. SH, MT, and KY provided the Ccn3-KO mice. HK, GPS, LR, GBA, SLG, and BP provided reagents and advice. JVM, PN, and JG contributed reagents, advised on AAA pathogenesis, and critically revised the manuscript. ND and ZL secured funding for the research. ZL wrote the manuscript. All authors discussed the results and edited the manuscript.

\section{Acknowledgments}

We thank Mukesh K. Jain for critical review of this manuscript. This work was supported by NIH grants HL117759 and AA021390 (to Z. Lin). This work was also supported in part by the National Nature Science Foundation of China (grant number 31330029).

Address correspondence to: Zhiyong Lin, Case Cardiovascular Research Institute, 2103 Cornell Road, Room 4-541, Cleveland, Ohio 44106, USA. Phone: 216.368.0767; E-mail: Zhiyong.lin@ case.edu.

Bernard Perbal's present address is: GREDEG, Université de Nice Sophia-Antipolis, France.
1. Weintraub NL. Understanding abdominal aortic aneurysm. N Engl J Med. 2009;361(11):1114-1116.

2. Boddy AM, Lenk GM, Lillvis JH, Nischan J, Kyo $\mathrm{Y}$, Kuivaniemi H. Basic research studies to understand aneurysm disease. Drug News Perspect. 2008;21(3):142-148.

3. LeMaire SA, Russell L. Epidemiology of thoracic aortic dissection. Nat Rev Cardiol. 2011;8(2):103-113.

4. Nordon IM, Hinchliffe RJ, Holt PJ, Loftus IM, Thompson MM. Review of current theories for abdominal aortic aneurysm pathogenesis. Vascular. 2009;17(5):253-263.

5. El-Hamamsy I, Yacoub MH. Cellular and molecular mechanisms of thoracic aortic aneurysms. Nat Rev Cardiol. 2009;6(12):771-786.

6. Krishna SM, Dear AE, Norman PE, Golledge J. Genetic and epigenetic mechanisms and their possible role in abdominal aortic aneurysm. Atherosclerosis. 2010;212(1):16-29.

7. Golledge J, Norman PE. Current status of medical management for abdominal aortic aneurysm. Atherosclerosis. 2011;217(1):57-63.

8. Hellenthal FA, Buurman WA, Wodzig WK, Schurink GW. Biomarkers of AAA progression. Nat Rev Cardiol. 2009;6(7):464-474.

9. Mouw JK, Ou G, Weaver VM. Extracellular matrix assembly: a multiscale deconstruction. Nat Rev Mol Cell Biol. 2014;15(12):771-785.

10. Humphrey JD, Dufresne ER, Schwartz MA. Mechanotransduction and extracellular matrix homeostasis. Nat Rev Mol Cell Biol. 2014;15(12):802-812.

11. Xu J, Shi GP. Vascular wall extracellular matrix proteins and vascular diseases. Biochim Biophys Acta. 2014;1842(11):2106-2119.

12. Yeger H, Perbal B. The CCN family of genes: a perspective on CCN biology and therapeutic potential. JCell Commun Signal. 2007;1(3-4):159-164.

13. Jun JI, Lau LF. Taking aim at the extracellular matrix: $\mathrm{CCN}$ proteins as emerging therapeutic targets. Nat Rev Drug Discov. 2011;10(12):945-963.

14. Chen CC, Lau LF. Functions and mechanisms of action of CCN matricellular proteins. Int J Biochem Cell Biol. 2009;41(4):771-783.

15. Joliot V, et al. Proviral rearrangements and overexpression of a new cellular gene (nov) in myeloblastosis-associated virus type 1-induced nephroblastomas. Mol Cell Biol. 1992;12(1):10-21.

16. Lin Z, et al. A novel role of CCN3 in regulating endothelial inflammation. J Cell Commun Signal. 2010;4(3):141-153.

17. Shimoyama T, et al. CCN3 inhibits neointimal hyperplasia through modulation of smooth muscle cell growth and migration. Arterioscler Thromb Vasc Biol. 2010;30(4):675-682.

18. Ellis PD, Chen Q, Barker PJ, Metcalfe JC, Kemp PR. Nov gene encodes adhesion factor for vascular smooth muscle cells and is dynamically regulated in response to vascular injury. Arterioscler Thromb Vasc Biol. 2000;20(8):1912-1919.

19. Lenk GM, Tromp G, Weinsheimer S, Gatalica $\mathrm{Z}$, Berguer R, Kuivaniemi H. Whole genome expression profiling reveals a significant role for immune function in human abdominal aortic aneurysms. BMC Genomics. 2007;8:237.

20. Pyo R, et al. Targeted gene disruption of matrix metalloproteinase-9 (gelatinase B) suppresses development of experimental abdominal aortic aneurysms. JClin Invest. 2000;105(11):1641-1649.

21. Rateri DL, Howatt DA, Moorleghen JJ, Charnigo $\mathrm{R}$, Cassis LA, Daugherty A. Prolonged infusion of angiotensin II in apoE(-/-) mice promotes macrophage recruitment with continued expansion of abdominal aortic aneurysm. Am J Pathol. 2011;179(3):1542-1548.

22. Moxon JV, et al. Proteomic and genomic analyses suggest the association of apolipoprotein $\mathrm{C} 1$ with abdominal aortic aneurysm. Proteomics Clin Appl. 2014;8(9-10):762-772.

23. Rush C, Nyara M, Moxon JV, Trollope A, Cullen $\mathrm{B}$, Golledge J. Whole genome expression analysis within the angiotensin II-apolipoprotein E deficient mouse model of abdominal aortic aneurysm. BMC Genomics. 2009;10:298.

24. Daugherty A, Manning MW, Cassis LA. Antagonism of AT2 receptors augments angiotensin II-induced abdominal aortic aneurysms and atherosclerosis. Br J Pharmacol. 2001;134(4):865-870.

25. Yoshioka K, et al. Endothelial PI3K-C2 $\alpha$, a class II PI3K, has an essential role in angiogenesis and vascular barrier function. Nat Med. 2012;18(10):1560-1569.

26. Shimizu K, Mitchell RN, Libby P. Inflammation and cellular immune responses in abdominal aortic aneurysms. Arterioscler Thromb Vasc Biol. 2006;26(5):987-994.

27. Choke E, et al. A review of biological factors implicated in abdominal aortic aneurysm rupture. Eur J Vasc Endovasc Surg. 2005;30(3):227-244.

28. McCormick ML, Gavrila D, Weintraub NL. Role of oxidative stress in the pathogenesis of abdominal aortic aneurysms. Arterioscler Thromb Vasc Biol. 2007;27(3):461-469.

29. Davis FM, Rateri DL, Daugherty A. Mechanisms of aortic aneurysm formation: translating preclinical studies into clinical therapies. Heart.
2014;100(19):1498-1505.

30. Yoshimura K, et al. Regression of abdominal aortic aneurysm by inhibition of c-Jun $\mathrm{N}$-terminal kinase. Nat Med. 2005;11(12):1330-1338.

31. Ghosh A, et al. The role of extracellular signalrelated kinase during abdominal aortic aneurysm formation. J Am Coll Surg. 2012;215(5):668-680.

32. Miyake T, et al. Regression of abdominal aortic aneurysms by simultaneous inhibition of nuclear factor $\kappa \mathrm{B}$ and ets in a rabbit model. Circ Res. 2007;101(11):1175-1184.

33. Liao M, Xu J, Clair AJ, Ehrman B, Graham LM, Eagleton MJ. Local and systemic alterations in signal transducers and activators of transcription (STAT) associated with human abdominal aortic aneurysms. J Surg Res. 2012;176(1):321-328.

34. Maegdefessel L, et al. Inhibition of microRNA$29 \mathrm{~b}$ reduces murine abdominal aortic aneurysm development. J Clin Invest. 2012;122(2):497-506.

35. Chen X, Lu H, Rateri DL, Cassis LA, Daugherty A. Conundrum of angiotensin II and TGF- $\beta$ interactions in aortic aneurysms. Curr Opin Pharmacol. 2013;13(2):180-185.

36. Wang Y, Krishna S, Walker PJ, Norman P, Golledge J. Transforming growth factor- $\beta$ and abdominal aortic aneurysms. Cardiovasc Pathol. 2013;22(2):126-132.

37. Moran CS, et al. Everolimus limits aortic aneurysm in the apolipoprotein $\mathrm{E}$-deficient mouse by downregulating C-C chemokine receptor 2 positive monocytes. Arterioscler Thromb Vasc Biol. 2013;33(4):814-821.

38. Hatano N, et al. Essential role for ERK2 mitogenactivated protein kinase in placental development. Genes Cells. 2003;8(11):847-856.

39. Maegdefessel L, et al. MicroRNA-21 blocks abdominal aortic aneurysm development and nicotine-augmented expansion. Sci Transl Med. 2012;4(122):122ra122.

40. Esteban V, et al. Regulator of calcineurin 1 mediates pathological vascular wall remodeling. J Exp Med. 2011;208(10):2125-2139.

41. Cao J, et al. Lentiviral-human heme oxygenase targeting endothelium improved vascular function in angiotensin II animal model of hypertension. Hum Gene Ther. 2011;22(3):271-282.

42. Qian HS, et al. Overexpression of PAI-1 prevents the development of abdominal aortic aneurysm in mice. Gene Ther. 2008;15(3):224-232.

43. Perbal B. CCN proteins: A centralized communication network. JCell Commun Signal. 2013;7(3):169-177.

44. Babic AM, Chen CC, Lau LF. Fisp12/mouse connective tissue growth factor mediates endothelial 
cell adhesion and migration through integrin av $\beta 3$, promotes endothelial cell survival, and induces angiogenesis in vivo. Mol Cell Biol. 1999;19(4):2958-2966.

45. Babic AM, Kireeva ML, Kolesnikova TV, Lau LF. CYR61, a product of a growth factor-inducible immediate early gene, promotes angiogenesis and tumor growth. Proc Natl Acad Sci U S A. 1998;95(11):6355-6360.

46. Lin CG, et al. CCN3 (NOV) is a novel angiogenic regulator of the CCN protein family. J Biol Chem. 2003;278(26):24200-24208.

47. Schober JM, et al. Identification of integrin $\alpha(M)$ $\beta(2)$ as an adhesion receptor on peripheral blood monocytes for Cyr61 (CCN1) and connective tissue growth factor (CCN2): immediate-early gene products expressed in atherosclerotic lesions. Blood. 2002;99(12):4457-4465.

48. Oemar BS, et al. Human connective tissue growth factor is expressed in advanced atherosclerotic lesions. Circulation. 1997;95(4):831-839.

49. Branchetti E, et al. Oxidative stress modulates vascular smooth muscle cell phenotype via CTGF in thoracic aortic aneurysm. Cardiovasc Res. 2013;100(2):316-324.

50. Liu H, et al. CCN4 regulates vascular smooth muscle cell migration and proliferation. Mol Cells. 2013;36(2):112-118.

51. Matsumae H, et al. CCN1 knockdown suppresses neointimal hyperplasia in a rat artery balloon injury model. Arterioscler Thromb Vasc Biol. 2008;28(6):1077-1083.

52. Grzeszkiewicz TM, Lindner V, Chen N, Lam
SC, Lau LF. The angiogenic factor cysteine-rich 61 (CYR61, CCN1) supports vascular smooth muscle cell adhesion and stimulates chemotaxis through integrin $\alpha(6) \beta(1)$ and cell surface heparan sulfate proteoglycans. Endocrinology. 2002;143(4):1441-1450.

53. Fan WH, Pech M, Karnovsky MJ. Connective tissue growth factor (CTGF) stimulates vascular smooth muscle cell growth and migration in vitro. Eur JCell Biol. 2000;79(12):915-923.

54 . Tsai SH, et al. Zoledronate attenuates angiotensin II-induced abdominal aortic aneurysm through inactivation of Rho/ROCK-dependent JNK and NF- $\mathrm{kB}$ pathway. Cardiovasc Res. 2013;100(3):501-510.

55. Wang YX, et al. Fasudil, a Rho-kinase inhibitor, attenuates angiotensin II-induced abdominal aortic aneurysm in apolipoprotein E-deficient mice by inhibiting apoptosis and proteolysis. Circulation. 2005;111(17):2219-2226.

56. Ghosh A, et al. Phosphorylation of AKT and abdominal aortic aneurysm formation. Am J Pathol. 2014;184(1):148-158.

57. Leeper NJ, et al. Loss of CDKN2B promotes p53-dependent smooth muscle cell apoptosis and aneurysm formation. Arterioscler Thromb Vasc Biol. 2013;33(1):e1-e10.

58. Haldar SM, et al. Klf15 deficiency is a molecular link between heart failure and aortic aneurysm formation. Sci Transl Med. 2010;2(26):26ra26.

59. Zou S, Ren P, Nguyen M, Coselli JS, Shen YH, LeMaire SA. Notch signaling in descending thoracic aortic aneurysm and dissection. PLoS One. 2012;7(12):e52833.

60. Wang S, et al. Activation of AMP-activated protein kinase $\alpha 2$ by nicotine instigates formation of abdominal aortic aneurysms in mice in vivo. Nat Med. 2012;18(6):902-910.

61. Shen $\mathrm{YH}$, et al. AKT2 confers protection against aortic aneurysms and dissections. Circ Res. 2013;112(4):618-632.

62. Gupta R, Hong D, Iborra F, Sarno S, Enver T. NOV (CCN3) functions as a regulator of human hematopoietic stem or progenitor cells. Science. 2007;316(5824):590-593.

63. McCallum L, Irvine AE. CCN3 - a key regulator of the hematopoietic compartment. Blood Rev. 2009;23(2):79-85.

64. Selcher JC, Nekrasova T, Paylor R, Landreth GE, Sweatt JD. Mice lacking the ERK1 isoform of MAP kinase are unimpaired in emotional learning. Learn Mem. 2001;8(1):11-19.

65. Daugherty A, Manning MW, Cassis LA. Angiotensin II promotes atherosclerotic lesions and aneurysms in apolipoprotein E-deficient mice. JClin Invest. 2000;105(11):1605-1612.

66. Chevalier G, et al. novH: differential expression in developing kidney and Wilm's tumors. Am J Pathol. 1998;152(6):1563-1575.

67. Schneider CA, Rasband WS, Eliceiri KW. NIH Image to ImageJ: 25 years of image analysis. Nat Methods. 2012;9(7):671-675.

68. Wang J, et al. IgE actions on $\mathrm{CD}^{+} \mathrm{T}$ cells, mast cells, and macrophages participate in the pathogenesis of experimental abdominal aortic aneurysms. EMBO Mol Med. 2014;6(7):952-969. 